\title{
Driver genes exome sequencing reveals distinct variants in African Americans with colorectal neoplasia
}

\author{
Hassan Ashktorab ${ }^{1}$, Hamed Azimi ${ }^{1}$, Sudhir Varma ${ }^{3}$, Edward L. Lee $^{2}$, Adeyinka 0. \\ Laiyemo ${ }^{1}$, Michael L. Nickerson ${ }^{4}$ and Hassan Brim ${ }^{2}$ \\ ${ }^{1}$ Department of Medicine, Cancer Center, Howard University, Washington, DC, USA \\ ${ }^{2}$ Department of Pathology, Howard University College of Medicine, Washington, DC, USA \\ ${ }^{3}$ Hithru Analytics, LLC, Silver Spring, MD, USA \\ ${ }^{4}$ Laboratory of Translational Genomics, National Cancer Institute, Bethesda, MD, USA \\ Correspondence to: Hassan Ashktorab, email: hashktorab@howard.edu \\ Keywords: colon; targeted sequencing; African Americans; actionable; druggable \\ Received: August 28, $2018 \quad$ Accepted: January 31, $2019 \quad$ Published: April 05, 2019 \\ Copyright: Ashktorab et al. This is an open-access article distributed under the terms of the Creative Commons Attribution License \\ 3.0 (CC BY 3.0), which permits unrestricted use, distribution, and reproduction in any medium, provided the original author and \\ source are credited.
}

\section{ABSTRACT}

Background: Colorectal cancer (CRC) is the third leading cause of cancer-related deaths in the United States. African Americans are disproportionately affected by CRC. Our hypothesis is that driver genes with known and novel mutations have an impact on CRC outcome in this population. Therefore, we investigated the variants' profiles in a panel of 15 CRC genes.

Patients \& Methods: Colorectal specimens $(n=140)$ were analyzed by targeted exome sequencing using an Ion Torrent platform. Detected variants were validated in 36 samples by Illumina sequencing. The novel status of the validated variants was determined by comparison to publicly available databases. Annotated using ANNOVAR and in-silico functional analysis of these variants were performed to determine likely pathogenic variants.

Results: Overall, 121 known and novel variants were validated: APC (27\%), AMER1 (3\%), ARID1 (7\%), MSH3 (12\%), MSH6 (10\%), BRAF (4\%), KRAS (6\%), FBXW7 (4\%), PIK3CA (6\%), SMAD4 (5\%), SOX9 (2\%), TCF7L2 (2\%), TGFBR2 (5\%), TP53 (7\%). From these validated variants, 12\% were novel in 8 genes (AMER1, APC, ARID1A, BRAF, MSH6, PIK3CA, SMAD4, and TCF7L2). Of the validated variants, $23 \%$ were non-synonymous, $14 \%$ were stopgains, $24 \%$ were synonymous and $39 \%$ were intronic variants.

Conclusion: We here report the specifics of variants' profiles of African Americans with colorectal lesions. Validated variants showed that Tumor Suppressor Genes (TSGs) APC and ARID1 and DNA Mismatch repair (MMR) genes MSH3 and MSH6 are the genes with the highest numbers of validated variants. Oncogenes KRAS and PIK3CA are also altered and likely participate in the increased proliferative potential of the mutated colonic epithelial cells in this population.

\section{INTRODUCTION}

Colorectal Cancer (CRC) is the third leading cause of cancer-related deaths in the US. Its incidence and mortality are higher in African Americans (AAs) than other ethnic groups in the US. The reasons for this disparity are not yet well established [1]. It is thought that the underlying factors behind this disparity are multiple (diet, lifestyle, microbiome, socioeconomic, healthcare access and genetic predispositions). Cancer driver mutations play an important role in the carcinogenic process $[2,3]$. There are limited next generation sequencing studies in African Americans 
with cancer in general and with CRC in particular [46]. However, none of these studies investigated cancer specimens along with pre-neoplastic lesions.

Molecularly, CRCs are categorized into those with microsatellite instability (MSI) which are located primarily in the right colon and frequently associate with the $\mathrm{CpG}$ island methylator phenotype (CIMP) and those that are microsatellite stable (MSS) but are chromosomally unstable [7-9]. MSI characterizes $10-15 \%$ of sporadic $\mathrm{CRCs}$ and has been related to a better prognosis compared with MSS colorectal cancer patients [7, 10]. Most MSI $\mathrm{CRCs}$ have been assigned previously to defects in $\mathrm{MLHI}$ and MSH2 genes within the DNA Mismatch Repair (MMR) genes machinery. However recent attention has been given to other genes such as MSH3 and MSH6 that were found to be altered and associated with a different category of microsatellite instability within the genome, primarily at tetranucleotide repeats [11].

Several pathogenic gene panels that are frequently mutated in CRC have been designed for targeted sequencing. We examined 15 genes associated with CRC using a Personal Genome Machine (PGM; Ion TorrentThermoFisher Scientific; Waltham, MA) sequencing platform for variant discovery, and a MiSeq (Illumina; San Diego, CA) sequencing platform for validation. The 15 genes correspond to two DNA MMR genes, 6 oncogenes and 7 tumor suppressor genes (TSGs). The DNA MMR gene MSH3 have acquired more attention recently in CRC patients as variants in this gene were found to be prevalent in African Americans (50 to 60\%) and associate with poor prognosis $[11,12]$ in contrast with MSI-H phenotype that is driven by altered $M L H 1$ and $M S H 2$ alterations. The 15 genes panel also consisted of 6 oncogenes namely: $B R A F$, NRAS, KRAS, PIK3CA, SMAD4 and SOX9. KRAS is involved in the pathogenesis of many different malignant tumors, including lung, pancreatic, and colon cancers [13]. Around 30 to $40 \%$ of CRCs have KRAS variants [14]. $N R A S$ is a member of a family of oncoproteins that are commonly mutated in cancer. Activating variants in NRAS occur in a subset of CRC but little is known about how the mutant protein contributes to the onset and progression of the disease [14]. BRAF is mutated in 4 to $12 \%$ of unselected $\mathrm{CRC}$, particularly those with high microsatellite instability [15]. BRAF mutations in CRC are associated with distinct clinical characteristics and a worse prognosis [16]. PIK3CA encodes for the catalytic p110-alpha subunit of Phosphatidylinositol 3-Kinase (PI3K) alpha, which orchestrates cell responses including cell proliferation, survival, migration and morphology [17]. Activating mutations in PIK3CA were reported in 10 to $15 \%$ of colorectal carcinomas [18]. SOX9 has been widely studied in the context of development and cell lineage determination in various tissues. Recent studies have indicated tissue- and context-specific roles of this gene [19].

The gene panel contained 7 tumor suppressor genes (AMER1, APC, ARID1, FBXW7, TCF7L2, TGFBR2, and
TP53). APC is one of the key genes in the initiation of polyp formation [20] in both familial adenomatous polyposis (FAP) and FAP-like sporadic CRCs [21]. Current studies have shown mutations of $A P C$ in many cancers including CRC. Several studies have suggested that chromosome $18 \mathrm{q}$ loss is a critical event during CRC progression and that the SMAD4 tumor suppressor is the primary target for inactivation [22]. Clinical studies have shown that patients retaining heterozygosity at the $18 \mathrm{q}$ locus benefit significantly better from treatment with 5-Fluorouracil than patients with loss of heterozygosity $(\mathrm{LOH})$ at this site [23]. AT-Rich Interactive Domain 1A (ARIDIA) has recently been identified as a novel tumor suppressor in various tumor types. Loss of ARIDIA expression is uncommon and not associated with oncologic outcome but may be related to less invasive pathologic features in CRC [24]. Most CRCs with microsatellite instability (MSI-H) have mutations in a microsatellite sequence encoding Transforming Growth Factor $\beta$ Receptor II (TGFBR2). Therefore, it is understood that TGFBR2 is defective in these tumors, even though CRC cells with TGFBR2 variants have been described to remain sensitive to TGF $\beta$ [25]. Resistance to growth inhibition by TGF $\beta$ is standard in a variety of human cancers, emphasizing the importance of intracellular pathways mediated by this polypeptide to the neoplastic process [26]. $F B X W 7$ variants occur in a variety of human cancers including CRC [27]. FBXW7 and TP53 are tumor suppressors intensively implicated in colorectal carcinogenesis [28]. FBXW7 constitutes one of the four subunits of SCF (SKP1-cullin-F-box)-E3 ubiquitinprotein ligase complex, which functions in phosphorylationdependent ubiquitination [27]. About half of all CRCs show TP53 gene variants, with lower frequencies in proximal tumors and higher frequencies in distal colon and rectal tumors and in those with the microsatellite instability or methylator phenotypes [28]. AMER1 regulates the distribution of the tumor suppressor APC between microtubules and the plasma membrane [29]. It is frequently mutated in colorectal cancer tumors [30]. TCF7L2 is a transcription factor of which polymorphisms have been associated with cancers including colon and prostate [31]. However, this gene's polymorphisms have been intensively studied in the context of diabetes-associated disorders [32].

In the present study, we examined and validated variants in a panel of cancer genes and assessed their association with disease characteristics in African Americans with colorectal neoplastic lesions.

\section{RESULTS}

\section{Clinical and pathological characteristics of patients}

A: Discovery set: The characteristics of the 123 patients from whom the 140 samples were collected are reported in Figure 1. The patients consisted of 69 (57\%) 
males. The age range at the time of diagnosis was 24 to 95 years, with a median of 61 years. With regard to cancer stage, $11 \%$ (6/56) were stage I, 34\% (19/56) were stage II, $29 \%(16 / 56)$ were stage III, and 4\% (2/56) were stage IV $(23 \%(13 / 56)$ had no staging data).

B: Validation set: Contained a subset of the discovery set and was made of 36 samples from 26 patients. There were $10(38 \%)$ females and $16(62 \%)$ males. With regard to cancer stage, $35 \%(9 / 26)$ were stage II, $15 \%(4 / 26)$ were stage III and $8 \%(2 / 26)$ stage IV, and $42 \%(11 / 26)$ had no staging data. The age range was from 41 to 88 with median age of 61 years (Table 1 ).

\section{Tumor suppressor genes}

\section{$A P C$ variants}

We detected 944 variants in the discovery set, of which 822 are novel. The detailed information of these variants was recently published [5]. The Illumina sequencing led to the validation of 33 variants of which 4 were novel. Variant at locus chr5:112176918 with a G to A change had a frequency 0.03 (1/33, heterozygous) in advanced adenoma (Amino acid change from $\mathrm{R}$ to T). Variant at locus chr5: 112174763 with an A to $\mathrm{T}$ change had a frequency of 0.02 (1/56, heterozygous) in CRCs (Amino acid change from $\mathrm{R}$ to $\mathrm{X}$ ). Variant at locus chr5:112154980 with a $\mathrm{T}$ to A change had a frequency
$0.05(1 / 21$, heterozygous) in adenoma (Amino acid change from $\mathrm{C}$ to $\mathrm{X}$ ). Variant at locus 112157658 with a $\mathrm{G}$ to $\mathrm{T}$ change had a frequency of $0.05(1 / 21$, heterozygous) in adenoma (Amino acid change from $\mathrm{E}$ to $\mathrm{X}$ ). One variant was mapped in the 5 , UTR, 9 prior to ARM, 4 on the $\mathrm{ARM}, 3$ prior to $\mathrm{B}-\mathrm{Catenin}$ binding region, 12 in the $\mathrm{B}-\mathrm{Catenin}$ binding region, 3 in the basic region, and 1 in the EB1 binding domain [5] (Table 2 and Figure 3A).

\section{AMER1 variants}

We found 461 variants in the discovery set of which 433 were novel. From these, 402 were non-synonymous, 25 were stopgains, and 6 were frameshift substitutions. All of these variants were in exon 2 of the AMER 1 gene. Four variants in the $A M E R 1$ gene were validated on Illumina HiSeq of which 1 was novel. This variant at locus chrX: 63409685 , a $\mathrm{C}$ to $\mathrm{T}$ change, had a frequency of 0.07 $(2 / 30$, both heterozygous) in normal tissue, $0.05(1 / 21$, heterozygous) in adenoma, 0.03 (1/33, heterozygous) in advanced adenoma and 0.02 (1/56, heterozygous) in CRC. (Table 2 and Figure 3B).

\section{ARIDIA variants}

We found 742 variants in the discovery set, of which 695 were novel. From these, 653 were non-synonymous, 35 were stopgains, 6 were frameshift and 1 was a non-frameshift variant. Eight variants in the $A R I D 1 A$ gene were validated on the HiSeq platform of which 2 were novel (Table 2).

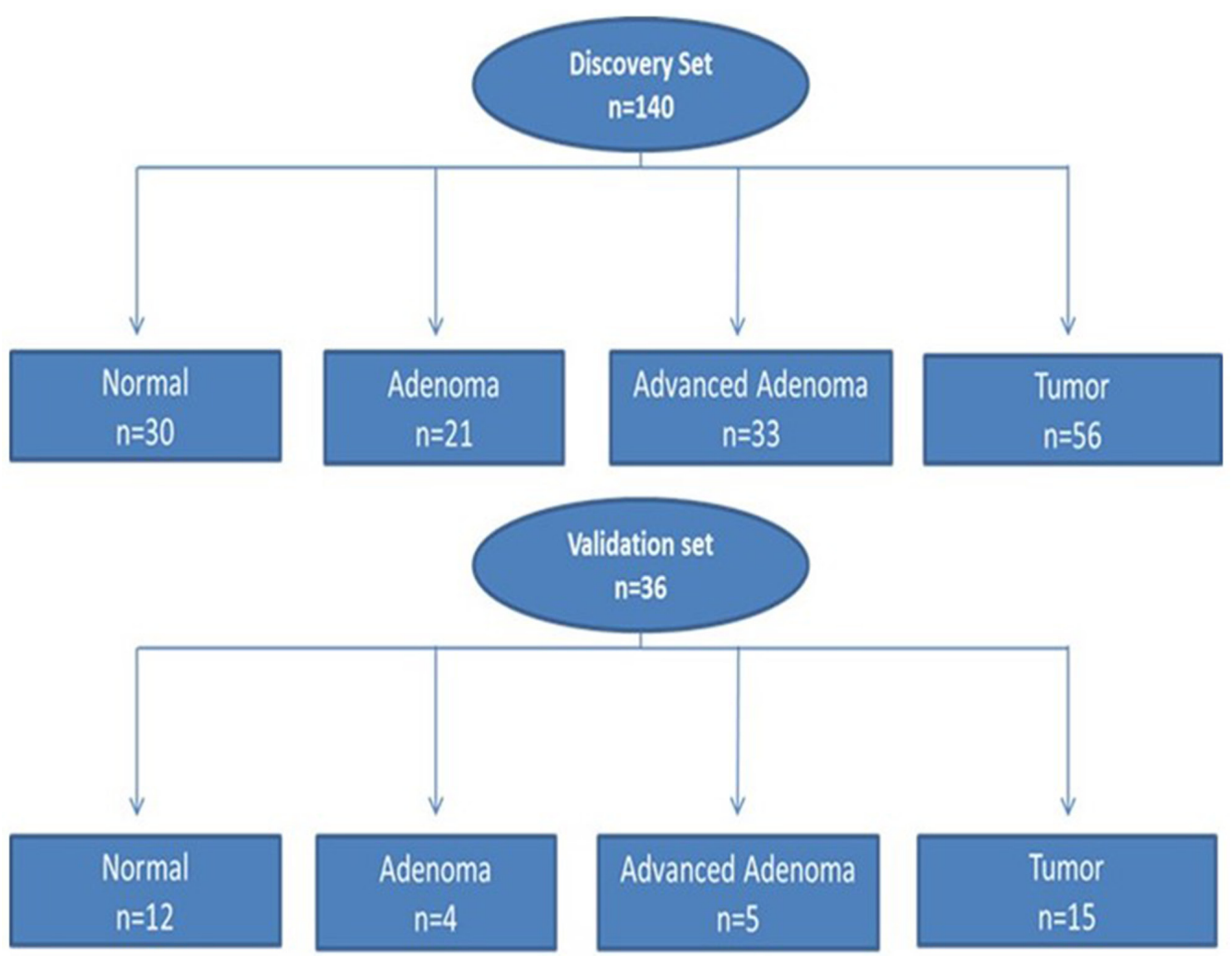

Figure 1: Flow chart of patient selection for both discovery and validation sets for somatic variants analysis. Discovery set: 140 samples $(n=123$ patients $)$ and validation set: 36 samples $(n=26$ patients $)$. 
Table 1: Clinico-pathological characteristics of the validation set (26 Patients \& 36 Samples)

\begin{tabular}{|c|c|c|c|c|c|c|}
\hline Sample ID & Age & Sex & Type of Tissue & Location & TNM & STAGING \\
\hline CC1018N & 84 & $\mathrm{~F}$ & $\mathrm{~N}$ & RIGHT & $\mathrm{N}$ & NA \\
\hline CC1018 & 84 & $\mathrm{~F}$ & $\mathrm{~T}$ & LEFT & $\mathrm{T} 3 \mathrm{~N} 1 \mathrm{~b}$ & II \\
\hline CC1024 & 73 & $\mathrm{~F}$ & $\mathrm{~T}$ & RIGHT & T3N1MX & III \\
\hline CC1028 & 42 & M & $\mathrm{T}$ & LEFT & T3N0MX & II \\
\hline $\mathrm{CC} 1028 \mathrm{~N}$ & 42 & M & $\mathrm{N}$ & RIGHT & $\mathrm{N}$ & NA \\
\hline CC1029 & 51 & $\mathrm{~F}$ & $\mathrm{~T}$ & RIGHT & T2N0MX & II \\
\hline CC1029N & 51 & $\mathrm{~F}$ & $\mathrm{~N}$ & LEFT & $\mathrm{N}$ & NA \\
\hline CC1036 & 63 & M & $\mathrm{T}$ & LEFT & T3N2M1 & IV \\
\hline $\mathrm{CC} 1036 \mathrm{~N}$ & 63 & M & $\mathrm{N}$ & RIGHT & $\mathrm{N}$ & NA \\
\hline CC1038 & 54 & M & $\mathrm{T}$ & LEFT & T3N0Mx & II \\
\hline CC1038N & 54 & M & $\mathrm{N}$ & RIGHT & $\mathrm{N}$ & NA \\
\hline CC1053 & 50 & $\mathrm{~F}$ & $\mathrm{~T}$ & RIGHT & T3N0M0 & II \\
\hline CC1053N & 50 & $\mathrm{~F}$ & $\mathrm{~N}$ & LEFT & $\mathrm{N}$ & NA \\
\hline CC1054 & 53 & $\mathrm{M}$ & $\mathrm{T}$ & RIGHT & T3N0M0 & II \\
\hline CC1054N & 53 & M & $\mathrm{N}$ & LEFT & $\mathrm{N}$ & NA \\
\hline CC1055 & 79 & $\mathrm{~F}$ & $\mathrm{AA}$ & RIGHT & $\mathrm{AA}$ & NA \\
\hline CC1056 & 66 & $\mathrm{M}$ & $\mathrm{T}$ & LEFT & T1N1MX & III \\
\hline CC1056N & 66 & $\mathrm{M}$ & $\mathrm{N}$ & RIGHT & $\mathrm{N}$ & NA \\
\hline CC1057 & 88 & $\mathrm{M}$ & $\mathrm{T}$ & LEFT & T3N2M0 & III \\
\hline CC1057N & 88 & $\mathrm{M}$ & $\mathrm{N}$ & RIGHT & $\mathrm{N}$ & NA \\
\hline CC1059 & 60 & $\mathrm{~F}$ & $\mathrm{~T}$ & RIGHT & T3N1MX & II \\
\hline CC1060 & 53 & $\mathrm{~F}$ & $\mathrm{~T}$ & LEFT & T3N2M1 & IV \\
\hline CC1060N & 53 & $\mathrm{~F}$ & $\mathrm{~N}$ & RIGHT & $\mathrm{N}$ & NA \\
\hline CC1061 & 63 & $\mathrm{M}$ & $\mathrm{N}$ & LEFT & $\mathrm{N}$ & NA \\
\hline $\mathrm{CC} 1065$ & 41 & M & $\mathrm{T}$ & LEFT & T3N0M0 & II \\
\hline CC1109 & 62 & $\mathrm{~F}$ & A & RIGHT & A & NA \\
\hline CC1258 & 52 & M & $\mathrm{T}$ & RIGHT & T3N1bMx & III \\
\hline CC1386 & 70 & $\mathrm{M}$ & $\mathrm{T}$ & LEFT & T3N0Mx & II \\
\hline CC1621N & 49 & M & $\mathrm{N}$ & RIGHT & $\mathrm{N}$ & NA \\
\hline CC1680 & 75 & $\mathrm{~F}$ & $\mathrm{AA}$ & RIGHT & AA & NA \\
\hline CC1681 & 75 & $\mathrm{M}$ & AA & LEFT & $\mathrm{AA}$ & NA \\
\hline CC1682 & 71 & M & $\mathrm{AA}$ & RIGHT & $\mathrm{AA}$ & NA \\
\hline CC1683 & 45 & $\mathrm{M}$ & A & RIGHT & AA & NA \\
\hline CC1698 & 54 & $\mathrm{M}$ & A & RIGHT & A & NA \\
\hline CC1720 & 70 & $\mathrm{~F}$ & A & RIGHT & A & NA \\
\hline CC1721 & 54 & $\mathrm{M}$ & A & Missing & $\mathrm{A}$ & NA \\
\hline
\end{tabular}

$\mathrm{N}=$ Normal, $\mathrm{T}=\mathrm{CRC}, \mathrm{A}=$ Adenoma, $\mathrm{AA}=$ Advanced Adenoma 
Table 2: Number of samples per validated variants in the targeted gene panel

\begin{tabular}{|c|c|c|c|c|c|c|c|c|c|c|c|c|c|}
\hline Locus & Ref & Var & Gene & Variant type & $\begin{array}{c}\text { Status } \\
(1=\text { novel, } \\
0=\text { known })\end{array}$ & $\begin{array}{c}\text { AA- } \\
\text { Normal } \\
\text { Het } \\
(30)\end{array}$ & $\begin{array}{c}\text { AA- } \\
\text { Normal } \\
\text { Hom } \\
(\mathbf{3 0})\end{array}$ & $\begin{array}{c}\text { AA- } \\
\text { Adenoma } \\
\text { Het (21) }\end{array}$ & $\begin{array}{c}\text { AA- } \\
\text { Adenoma } \\
\text { Hom } \\
(21)\end{array}$ & $\begin{array}{c}\text { AA-Ad. } \\
\text { Adenoma } \\
\text { Het (33) }\end{array}$ & $\begin{array}{c}\text { AA-Ad. } \\
\text { Adenoma } \\
\text { Hom (33) }\end{array}$ & $\begin{array}{c}\text { AA- } \\
\text { CRC } \\
\text { Het } \\
(56)\end{array}$ & $\begin{array}{l}\text { AA- } \\
\text { CRC } \\
\text { Hom } \\
(\mathbf{5 6 )}\end{array}$ \\
\hline 63409685 & $\mathrm{C}$ & $\mathrm{T}$ & AMER1 & intronic & 1 & 2 & 0 & 1 & 0 & 1 & 0 & 1 & 0 \\
\hline 63410110 & $\mathrm{~T}$ & $\mathrm{C}$ & AMER1 & synonymous SNV & 0 & 8 & 16 & 10 & 9 & 9 & 13 & 8 & 33 \\
\hline 63412291 & $\mathrm{C}$ & G & AMER1 & non-synonymous SNV & 0 & 2 & 1 & 3 & 0 & 0 & 0 & 4 & 1 \\
\hline 63412690 & $\mathrm{~A}$ & $\mathrm{C}$ & AMER1 & non-synonymous SNV & 0 & 13 & 6 & 9 & 2 & 13 & 2 & 27 & 10 \\
\hline 112043384 & $\mathrm{~T}$ & G & $\mathrm{APC}$ & intronic & 0 & 6 & 0 & 3 & 0 & 7 & 1 & 12 & 0 \\
\hline 112103015 & $\mathrm{C}$ & A & APC & stopgain & 0 & 0 & 0 & 0 & 0 & 0 & 0 & 1 & 0 \\
\hline 112116592 & $\mathrm{C}$ & $\mathrm{T}$ & APC & stopgain & 0 & 0 & 0 & 0 & 0 & 0 & 0 & 2 & 0 \\
\hline 112128191 & $\mathrm{C}$ & $\mathrm{T}$ & APC & stopgain & 0 & 0 & 0 & 1 & 0 & 2 & 0 & 2 & 0 \\
\hline 112136947 & A & $\mathrm{T}$ & APC & intronic & 0 & 3 & 0 & 2 & 0 & 3 & 0 & 3 & 0 \\
\hline 112151261 & $\mathrm{C}$ & $\mathrm{T}$ & APC & stopgain & 0 & 0 & 0 & 0 & 0 & 1 & 0 & 1 & 0 \\
\hline 112154942 & $\mathrm{C}$ & $\mathrm{T}$ & APC & stopgain & 0 & 0 & 0 & 1 & 0 & 1 & 0 & 1 & 0 \\
\hline 112154980 & $\mathrm{~T}$ & A & $\mathrm{APC}$ & stopgain & 1 & 0 & 0 & 1 & 0 & 0 & 0 & 0 & 0 \\
\hline 112157658 & G & $\mathrm{T}$ & APC & stopgain & 1 & 0 & 0 & 1 & 0 & 0 & 0 & 0 & 0 \\
\hline 112162854 & $\mathrm{~T}$ & $\mathrm{C}$ & APC & synonymous SNV & 0 & 11 & 1 & 7 & 2 & 14 & 2 & 17 & 6 \\
\hline 112164561 & G & A & $\mathrm{APC}$ & synonymous SNV & 0 & 16 & 5 & 14 & 6 & 20 & 7 & 27 & 16 \\
\hline 112173553 & $\mathrm{~T}$ & G & $\mathrm{APC}$ & synonymous SNV & 0 & 1 & 0 & 1 & 0 & 0 & 0 & 0 & 0 \\
\hline 112173899 & $\mathrm{C}$ & $\mathrm{T}$ & APC & non-synonymous SNV & 0 & 1 & 0 & 1 & 0 & 2 & 0 & 3 & 0 \\
\hline 112174096 & $\mathrm{C}$ & A & $\mathrm{APC}$ & stopgain & 0 & 0 & 0 & 0 & 0 & 1 & 0 & 0 & 0 \\
\hline 112174763 & A & $\mathrm{T}$ & $\mathrm{APC}$ & stopgain & 1 & 0 & 0 & 0 & 0 & 0 & 0 & 1 & 0 \\
\hline 112175023 & $\mathrm{~A}$ & G & APC & synonymous SNV & 0 & 3 & 0 & 2 & 0 & 4 & 0 & 3 & 0 \\
\hline 112175030 & G & $\mathrm{A}$ & APC & non-synonymous SNV & 0 & 1 & 0 & 0 & 0 & 0 & 0 & 2 & 0 \\
\hline 112175069 & $\mathrm{C}$ & $\mathrm{T}$ & $\mathrm{APC}$ & stopgain & 0 & 0 & 0 & 1 & 0 & 0 & 0 & 2 & 0 \\
\hline 112175207 & G & $\mathrm{T}$ & APC & stopgain & 0 & 0 & 0 & 0 & 0 & 0 & 0 & 2 & 0 \\
\hline 112175399 & A & $\mathrm{T}$ & $\mathrm{APC}$ & stopgain & 0 & 0 & 0 & 0 & 0 & 2 & 0 & 1 & 0 \\
\hline 112175576 & $\mathrm{C}$ & $\mathrm{T}$ & APC & stopgain & 0 & 0 & 0 & 0 & 0 & 3 & 0 & 0 & 0 \\
\hline 112175639 & $\mathrm{C}$ & $\mathrm{T}$ & APC & stopgain & 0 & 0 & 0 & 2 & 0 & 4 & 0 & 2 & 0 \\
\hline 112175770 & G & $\mathrm{A}$ & $\mathrm{APC}$ & synonymous SNV & 0 & 17 & 5 & 13 & 7 & 20 & 8 & 26 & 14 \\
\hline 112176325 & G & A & APC & synonymous SNV & 0 & 19 & 4 & 14 & 6 & 21 & 6 & 26 & 17 \\
\hline 112176541 & $\mathrm{C}$ & G & APC & synonymous SNV & 0 & 1 & 0 & 1 & 0 & 0 & 0 & 2 & 0 \\
\hline 112176559 & $\mathrm{~T}$ & G & $\mathrm{APC}$ & synonymous SNV & 0 & 18 & 4 & 13 & 7 & 19 & 8 & 26 & 17 \\
\hline 112176756 & $\mathrm{~T}$ & A & $\mathrm{APC}$ & non-synonymous SNV & 0 & 3 & 26 & 4 & 17 & 8 & 24 & 4 & 50 \\
\hline 112176918 & G & $\mathrm{C}$ & APC & non-synonymous SNV & 1 & 0 & 0 & 0 & 0 & 1 & 0 & 0 & 0 \\
\hline 112177171 & G & A & APC & synonymous SNV & 0 & 16 & 5 & 14 & 6 & 21 & 7 & 31 & 15 \\
\hline 112178492 & $\mathrm{C}$ & $\mathrm{T}$ & $\mathrm{APC}$ & synonymous SNV & 0 & 0 & 0 & 0 & 0 & 1 & 0 & 1 & 0 \\
\hline 112178795 & G & A & APC & non-synonymous SNV & 0 & 1 & 0 & 0 & 0 & 0 & 0 & 2 & 0 \\
\hline 112178995 & $\mathrm{~A}$ & G & APC & synonymous SNV & 0 & 7 & 0 & 3 & 0 & 6 & 0 & 6 & 2 \\
\hline
\end{tabular}




\begin{tabular}{|c|c|c|c|c|c|c|c|c|c|c|c|c|c|}
\hline Locus & Ref & Var & Gene & Variant type & $\begin{array}{c}\text { Status } \\
(1=\text { novel, } \\
0=\text { known })\end{array}$ & $\begin{array}{c}\text { AA- } \\
\text { Normal } \\
\text { Het } \\
(\mathbf{3 0})\end{array}$ & $\begin{array}{c}\text { AA- } \\
\text { Normal } \\
\text { Hom } \\
(\mathbf{3 0})\end{array}$ & $\begin{array}{c}\text { AA- } \\
\text { Adenoma } \\
\text { Het (21) }\end{array}$ & $\begin{array}{c}\text { AA- } \\
\text { Adenoma } \\
\text { Hom } \\
\text { (21) }\end{array}$ & $\begin{array}{c}\text { AA-Ad. } \\
\text { Adenoma } \\
\text { Het (33) }\end{array}$ & $\begin{array}{c}\text { AA-Ad. } \\
\text { Adenoma } \\
\text { Hom (33) }\end{array}$ & $\begin{array}{l}\text { AA- } \\
\text { CRC } \\
\text { Het } \\
(56)\end{array}$ & $\begin{array}{l}\text { AA- } \\
\text { CRC } \\
\text { Hom } \\
(56)\end{array}$ \\
\hline 112179909 & $\mathrm{C}$ & A & $\mathrm{APC}$ & intronic & 0 & 9 & 1 & 9 & 1 & 12 & 0 & 20 & 3 \\
\hline 27057621 & $\mathrm{~A}$ & $\mathrm{C}$ & ARID1A & intronic & 0 & 3 & 0 & 0 & 0 & 8 & 0 & 0 & 0 \\
\hline 27089446 & G & $\mathrm{C}$ & ARID1A & intronic & 0 & 2 & 0 & 0 & 0 & 5 & 1 & 1 & 0 \\
\hline 27089585 & $\mathrm{C}$ & A & ARID1A & synonymous SNV & 0 & 0 & 0 & 1 & 0 & 1 & 0 & 0 & 0 \\
\hline 27101278 & $\mathrm{C}$ & $\mathrm{T}$ & ARID1A & synonymous SNV & 0 & 1 & 0 & 0 & 0 & 0 & 0 & 1 & 0 \\
\hline 27102075 & G & A & ARID1A & synonymous SNV & 0 & 2 & 0 & 2 & 0 & 0 & 0 & 2 & 0 \\
\hline 27102188 & $\mathrm{~A}$ & G & ARID1A & non-synonymous SNV & 0 & 1 & 0 & 4 & 0 & 7 & 0 & 7 & 0 \\
\hline 27105676 & G & $\mathrm{T}$ & ARID1A & stopgain & 1 & 0 & 0 & 0 & 0 & 0 & 0 & 1 & 0 \\
\hline 27106323 & $\mathrm{C}$ & $\mathrm{T}$ & ARID1A & synonymous SNV & 1 & 1 & 0 & 0 & 0 & 0 & 0 & 4 & 0 \\
\hline 140434597 & G & $\mathrm{A}$ & BRAF & intronic & 0 & 7 & 0 & 4 & 0 & 6 & 0 & 5 & 0 \\
\hline 140449071 & $\mathrm{C}$ & $\mathrm{G}$ & BRAF & intronic & 0 & 7 & 15 & 9 & 7 & 20 & 8 & 28 & 18 \\
\hline 140449150 & $\mathrm{~T}$ & $\mathrm{C}$ & BRAF & synonymous SNV & 0 & 10 & 13 & 11 & 5 & 20 & 8 & 30 & 18 \\
\hline 140481511 & $\mathrm{~A}$ & $\mathrm{C}$ & BRAF & intronic & 0 & 2 & 0 & 2 & 0 & 0 & 0 & 3 & 0 \\
\hline 140487360 & G & A & BRAF & non-synonymous SNV & 1 & 0 & 0 & 0 & 0 & 0 & 0 & 1 & 0 \\
\hline 79950497 & $\mathrm{C}$ & $\mathrm{T}$ & DHFR,MSH3 & intronic & 0 & 6 & 4 & 6 & 2 & 3 & 2 & 14 & 5 \\
\hline 79950508 & $\mathrm{C}$ & $\mathrm{T}$ & DHFR,MSH3 & intronic & 0 & 4 & 0 & 1 & 0 & 3 & 0 & 5 & 0 \\
\hline 79950512 & A & G & DHFR,MSH3 & intronic & 0 & 5 & 14 & 1 & 8 & 3 & 8 & 13 & 26 \\
\hline 153245446 & G & A & FBXW7 & non-synonymous SNV & 0 & 0 & 0 & 0 & 0 & 1 & 0 & 0 & 0 \\
\hline 153247138 & G & $\mathrm{T}$ & FBXW7 & intronic & 0 & 1 & 0 & 0 & 0 & 0 & 0 & 1 & 0 \\
\hline 153247289 & G & A & FBXW7 & non-synonymous SNV & 0 & 0 & 0 & 0 & 0 & 1 & 0 & 2 & 0 \\
\hline 153247366 & $\mathrm{C}$ & $\mathrm{T}$ & FBXW7 & non-synonymous SNV & 0 & 0 & 0 & 0 & 0 & 1 & 0 & 0 & 0 \\
\hline 153303509 & $\mathrm{C}$ & $\mathrm{T}$ & FBXW7 & intronic & 0 & 1 & 0 & 0 & 0 & 1 & 0 & 1 & 0 \\
\hline 25362854 & $\mathrm{C}$ & $\mathrm{T}$ & KRAS & intronic & 0 & 11 & 3 & 12 & 0 & 10 & 0 & 24 & 5 \\
\hline 25368462 & $\mathrm{C}$ & $\mathrm{T}$ & KRAS & synonymous SNV & 0 & 0 & 29 & 0 & 21 & 1 & 32 & 1 & 53 \\
\hline 25398255 & G & $\mathrm{T}$ & KRAS & non-synonymous SNV & 0 & 0 & 0 & 0 & 0 & 1 & 0 & 0 & 0 \\
\hline 25398281 & $\mathrm{C}$ & $\mathrm{T}$ & KRAS & non-synonymous SNV & 0 & 0 & 0 & 3 & 0 & 5 & 0 & 9 & 0 \\
\hline 25398284 & $\mathrm{C}$ & $\mathrm{T}$ & KRAS & non-synonymous SNV & 0 & 0 & 0 & 3 & 0 & 2 & 0 & 12 & 0 \\
\hline 25398285 & $\mathrm{C}$ & A & KRAS & non-synonymous SNV & 0 & 0 & 0 & 0 & 0 & 2 & 0 & 1 & 0 \\
\hline 25398285 & $\mathrm{C}$ & $\mathrm{T}$ & KRAS & non-synonymous SNV & 0 & 0 & 0 & 0 & 0 & 3 & 0 & 2 & 0 \\
\hline 79960955 & G & $\mathrm{A}$ & MSH3 & intronic & 0 & 11 & 5 & 10 & 4 & 16 & 1 & 24 & 7 \\
\hline 79966029 & G & A & MSH3 & synonymous SNV & 0 & 6 & 1 & 11 & 0 & 5 & 2 & 16 & 0 \\
\hline 79966197 & G & A & MSH3 & intronic & 0 & 11 & 5 & 11 & 4 & 19 & 1 & 26 & 5 \\
\hline 79968496 & $\mathrm{C}$ & $\mathrm{T}$ & MSH3 & intronic & 0 & 5 & 3 & 3 & 3 & 11 & 1 & 13 & 3 \\
\hline 80024685 & $\mathrm{C}$ & A & MSH3 & non-synonymous SNV & 0 & 0 & 0 & 2 & 0 & 0 & 0 & 0 & 0 \\
\hline 80024738 & A & G & MSH3 & non-synonymous SNV & 0 & 0 & 0 & 0 & 0 & 2 & 0 & 0 & 0 \\
\hline 80024783 & G & A & MSH3 & non-synonymous SNV & 0 & 1 & 0 & 0 & 0 & 5 & 0 & 2 & 0 \\
\hline 80083459 & G & A & MSH3 & synonymous SNV & 0 & 1 & 0 & 0 & 0 & 2 & 0 & 1 & 0 \\
\hline
\end{tabular}




\begin{tabular}{|c|c|c|c|c|c|c|c|c|c|c|c|c|c|}
\hline Locus & Ref & Var & Gene & Variant type & $\begin{array}{c}\text { Status } \\
(1=\text { novel, } \\
\text { 0=known) }\end{array}$ & $\begin{array}{c}\text { AA- } \\
\text { Normal } \\
\text { Het } \\
(30)\end{array}$ & $\begin{array}{c}\text { AA- } \\
\text { Normal } \\
\text { Hom } \\
(\mathbf{3 0})\end{array}$ & $\begin{array}{c}\text { AA- } \\
\text { Adenoma } \\
\text { Het (21) }\end{array}$ & $\begin{array}{c}\text { AA- } \\
\text { Adenoma } \\
\text { Hom } \\
(21)\end{array}$ & $\begin{array}{l}\text { AA-Ad. } \\
\text { Adenoma } \\
\text { Het (33) }\end{array}$ & $\begin{array}{l}\text { AA-Ad. } \\
\text { Adenoma } \\
\text { Hom (33) }\end{array}$ & $\begin{array}{l}\text { AA- } \\
\text { CRC } \\
\text { Het } \\
(56)\end{array}$ & $\begin{array}{l}\text { AA- } \\
\text { CRC } \\
\text { Hom } \\
(56)\end{array}$ \\
\hline 80149981 & $\mathrm{~A}$ & G & MSH3 & non-synonymous SNV & 0 & 2 & 27 & 4 & 16 & 7 & 23 & 5 & 48 \\
\hline 80160610 & $\mathrm{~T}$ & A & MSH3 & intronic & 0 & 5 & 2 & 4 & 0 & 4 & 1 & 12 & 1 \\
\hline 80168937 & G & A & MSH3 & non-synonymous SNV & 0 & 11 & 15 & 10 & 8 & 19 & 10 & 22 & 30 \\
\hline 48022981 & G & $\mathrm{T}$ & MSH6 & intronic & 0 & 10 & 3 & 12 & 1 & 11 & 1 & 23 & 4 \\
\hline 48023115 & $\mathrm{~T}$ & $\mathrm{C}$ & MSH6 & synonymous SNV & 0 & 9 & 1 & 5 & 1 & 7 & 1 & 14 & 1 \\
\hline 48026286 & $\mathrm{C}$ & $\mathrm{T}$ & MSH6 & synonymous SNV & 0 & 4 & 0 & 2 & 0 & 4 & 0 & 7 & 0 \\
\hline 48027375 & $\mathrm{~T}$ & $\mathrm{C}$ & MSH6 & synonymous SNV & 0 & 4 & 0 & 2 & 0 & 7 & 0 & 5 & 1 \\
\hline 48030692 & $\mathrm{~T}$ & A & MSH6 & synonymous SNV & 0 & 0 & 0 & 0 & 0 & 1 & 0 & 0 & 0 \\
\hline 48030838 & A & $\mathrm{T}$ & MSH6 & intronic & 0 & 10 & 2 & 12 & 0 & 8 & 1 & 17 & 2 \\
\hline 48032908 & A & $\mathrm{G}$ & MSH6 & intronic & 1 & 1 & 0 & 1 & 0 & 0 & 0 & 2 & 0 \\
\hline 48032937 & $\mathrm{~T}$ & $\mathrm{C}$ & MSH6 & intronic & 0 & 4 & 25 & 4 & 17 & 9 & 21 & 12 & 40 \\
\hline 48033514 & $\mathrm{~T}$ & $\mathrm{C}$ & MSH6 & intronic & 0 & 0 & 0 & 2 & 0 & 2 & 0 & 1 & 0 \\
\hline 48033545 & A & $\mathrm{C}$ & MSH6 & intronic & 1 & 0 & 0 & 0 & 0 & 0 & 0 & 1 & 0 \\
\hline 48033551 & $\mathrm{C}$ & G & MSH6 & intronic & 0 & 4 & 20 & 3 & 11 & 8 & 9 & 13 & 34 \\
\hline 48033700 & G & A & MSH6 & non-synonymous SNV & 0 & 1 & 0 & 2 & 0 & 0 & 0 & 2 & 0 \\
\hline 178917005 & $\mathrm{~A}$ & G & PIK3CA & intronic & 0 & 8 & 7 & 11 & 2 & 16 & 3 & 28 & 7 \\
\hline 178921639 & $\mathrm{C}$ & A & PIK3CA & intronic & 0 & 12 & 16 & 11 & 9 & 15 & 14 & 24 & 25 \\
\hline 178922274 & $\mathrm{C}$ & A & PIK3CA & intronic & 0 & 6 & 17 & 11 & 9 & 8 & 14 & 14 & 26 \\
\hline 178927345 & $\mathrm{~T}$ & $\mathrm{C}$ & PIK3CA & intronic & 0 & 5 & 1 & 5 & 0 & 5 & 0 & 9 & 1 \\
\hline 178927410 & $\mathrm{~A}$ & G & PIK3CA & non-synonymous SNV & 0 & 4 & 2 & 7 & 0 & 12 & 2 & 23 & 1 \\
\hline 178947985 & $\mathrm{C}$ & G & PIK3CA & intronic & 1 & 0 & 0 & 0 & 0 & 0 & 0 & 1 & 0 \\
\hline 178948196 & A & G & PIK3CA & intronic & 0 & 6 & 0 & 4 & 1 & 4 & 0 & 6 & 0 \\
\hline 48575700 & $\mathrm{~T}$ & $\mathrm{C}$ & SMAD4 & intronic & 1 & 0 & 0 & 0 & 0 & 0 & 0 & 1 & 0 \\
\hline 48584420 & $\mathrm{G}$ & A & SMAD4 & intronic & 0 & 3 & 0 & 1 & 0 & 1 & 0 & 3 & 0 \\
\hline 48584624 & $\mathrm{~T}$ & $\mathrm{C}$ & SMAD4 & intronic & 1 & 0 & 0 & 0 & 0 & 0 & 0 & 1 & 0 \\
\hline 48591762 & G & A & SMAD4 & intronic & 0 & 1 & 0 & 9 & 1 & 7 & 0 & 9 & 0 \\
\hline 48592020 & $\mathrm{~T}$ & $\mathrm{C}$ & SMAD4 & intronic & 0 & 2 & 0 & 1 & 0 & 1 & 0 & 3 & 0 \\
\hline 48602941 & G & $\mathrm{C}$ & SMAD4 & intronic & 0 & 1 & 0 & 4 & 0 & 0 & 0 & 1 & 0 \\
\hline 70117450 & G & A & SOX9 & intronic & 0 & 3 & 0 & 1 & 0 & 0 & 1 & 3 & 1 \\
\hline 70118935 & $\mathrm{C}$ & $\mathrm{T}$ & SOX9 & synonymous SNV & 0 & 10 & 0 & 3 & 0 & 3 & 0 & 16 & 1 \\
\hline 70120551 & A & $\mathrm{C}$ & SOX9 & intronic & 0 & 13 & 1 & 8 & 0 & 11 & 3 & 19 & 0 \\
\hline 114912081 & G & $\mathrm{A}$ & TCF7L2 & intronic & 0 & 1 & 0 & 2 & 0 & 2 & 0 & 4 & 0 \\
\hline 114920452 & $\mathrm{~T}$ & $\mathrm{C}$ & TCF7L2 & intronic & 1 & 0 & 0 & 0 & 0 & 2 & 0 & 0 & 0 \\
\hline 30686414 & A & G & TGFBR2 & intronic & 0 & 11 & 4 & 10 & 3 & 16 & 4 & 23 & 5 \\
\hline 30713126 & $\mathrm{~T}$ & A & TGFBR2 & intronic & 0 & 6 & 0 & 10 & 0 & 12 & 0 & 13 & 1 \\
\hline 30713619 & $\mathrm{C}$ & $\mathrm{T}$ & TGFBR2 & non-synonymous SNV & 0 & 0 & 0 & 0 & 0 & 2 & 0 & 0 & 0 \\
\hline 30713674 & $\mathrm{~A}$ & G & TGFBR2 & synonymous SNV & 0 & 6 & 0 & 3 & 0 & 3 & 0 & 10 & 0 \\
\hline 30713842 & $\mathrm{C}$ & $\mathrm{T}$ & TGFBR2 & synonymous SNV & 0 & 2 & 0 & 1 & 0 & 2 & 0 & 2 & 0 \\
\hline \multirow[t]{2}{*}{30729931} & $\mathrm{C}$ & $\mathrm{T}$ & TGFBR2 & synonymous SNV & 0 & 2 & 0 & 0 & 0 & 0 & 0 & 2 & 0 \\
\hline & & & & & & & & & & & & \multicolumn{2}{|c|}{ (Continued) } \\
\hline
\end{tabular}




\begin{tabular}{|c|c|c|c|c|c|c|c|c|c|c|c|c|c|}
\hline Locus & Ref & Var & Gene & Variant type & $\begin{array}{c}\text { Status } \\
(1=\text { novel, } \\
0=\text { known })\end{array}$ & $\begin{array}{c}\text { AA- } \\
\text { Normal } \\
\text { Het } \\
(\mathbf{3 0})\end{array}$ & $\begin{array}{c}\text { AA- } \\
\text { Normal } \\
\text { Hom } \\
(\mathbf{3 0})\end{array}$ & $\begin{array}{c}\text { AA- } \\
\text { Adenoma } \\
\text { Het (21) }\end{array}$ & $\begin{array}{c}\text { AA- } \\
\text { Adenoma } \\
\text { Hom } \\
\text { (21) }\end{array}$ & $\begin{array}{c}\text { AA-Ad. } \\
\text { Adenoma } \\
\text { Het (33) }\end{array}$ & $\begin{array}{c}\text { AA-Ad. } \\
\text { Adenoma } \\
\text { Hom (33) }\end{array}$ & $\begin{array}{c}\text { AA- } \\
\text { CRC } \\
\text { Het } \\
(56)\end{array}$ & $\begin{array}{l}\text { AA- } \\
\text { CRC } \\
\text { Hom } \\
(56)\end{array}$ \\
\hline 7574003 & G & A & TP53 & stopgain & 0 & 0 & 0 & 0 & 0 & 1 & 0 & 1 & 0 \\
\hline 7576501 & G & $\mathrm{A}$ & TP53 & intronic & 0 & 1 & 0 & 0 & 0 & 2 & 0 & 5 & 0 \\
\hline 7577120 & $\mathrm{C}$ & $\mathrm{T}$ & TP53 & non-synonymous SNV & 0 & 0 & 0 & 0 & 0 & 2 & 0 & 2 & 0 \\
\hline 7578210 & $\mathrm{~T}$ & $\mathrm{C}$ & TP53 & synonymous SNV & 0 & 0 & 0 & 1 & 0 & 0 & 0 & 1 & 0 \\
\hline 7578212 & G & $\mathrm{A}$ & TP53 & stopgain & 0 & 0 & 0 & 0 & 0 & 0 & 0 & 4 & 0 \\
\hline 7578406 & $\mathrm{C}$ & $\mathrm{T}$ & TP53 & non-synonymous SNV & 0 & 1 & 0 & 0 & 0 & 0 & 0 & 3 & 0 \\
\hline 7579311 & $\mathrm{C}$ & A & TP53 & intronic & 0 & 0 & 0 & 0 & 0 & 0 & 0 & 1 & 0 \\
\hline 7579472 & G & $\mathrm{C}$ & TP53 & non-synonymous SNV & 0 & 21 & 0 & 14 & 0 & 22 & 0 & 34 & 0 \\
\hline 7579801 & G & $\mathrm{C}$ & TP53 & intronic & 0 & 23 & 0 & 12 & 1 & 20 & 0 & 36 & 0 \\
\hline
\end{tabular}

Variant at locus chr2: 27106323, was synonymous, a C to $\mathrm{T}$ change, with a frequency of $0.03(1 / 30$, heterozygous) in normal and 0.07 (4/56, all heterozygous) in CRCs. Variant at locus chr2: 27105676 was a stopgain, a $\mathrm{G}$ to $\mathrm{T}$ change, with a frequency of 0.02 (1/56, heterozygous) in CRCs (Amino acid change from $\mathrm{E}$ to $\mathrm{X}$ ). These variants were on exon 19 and 20 of the ARID1A, respectively. Three variants were mapped to $\mathrm{N}$-terminus sequences before the ARID domain, 1 prior to the GR [the C-terminus of the protein can stimulate Glucocorticoid Receptor-dependent transcriptional activation] binding domain, and 4 in the GR binding domain (Table 2 and Figure 3C).

\section{$F B X W 7$ variants}

We found 262 variants in the discovery set, of which 230 were novel. From these, there were 210 nonsynonymous and 20 stopgains. We validated five known variants. From these five, 2 were flanking intronic, and 3 were non-synonymous. Variant at locus Chr4: 153247289, exon 9, codon change of CGC to CAC had a frequency of $0.03(1 / 33$, heterozygous) in advanced adenoma and 0.04 (2/56, heterozygous) in CRCs, this variant was non-synonymous (Table 2). Non-synonymous variant at locus Chr4: 153247366, exon 9, a C to T change, had a frequency of 0.03 (1/33, heterozygous) in advanced adenoma. Variant at locus Chr4: 153245446, exon 10, codon change CAG to CAA, had a frequency of 0.03 $(1 / 33$, heterozygous) in advanced adenoma (Amino acid change from $\mathrm{S}$ to $\mathrm{L}$ ), this variant was non-synonymous (Table 2). Four variants were mapped to the WD repeat domain while 1 was prior to the $\mathrm{D}$ domain (Figure 3D).

\section{TCF7L2 variants}

We found 194 variants in the discovery set, of which 186 were novel. From these, 178 were non-synonymous, 5 were stopgains, 2 were frameshift substitutions, and 1 was stoploss. We validated two of these variants: 1 was novel flanking intronic at locus Chr10: 114920452, a T to C change, with a frequency of 0.06 ( $2 / 33$, both heterozygous) in advanced adenoma. The other variant at locus Chr10: 114912081 was known, a $\mathrm{G}$ to A change, had a frequency of $0.03(1 / 30$, heterozygous) in normal tissue, $0.10(2 / 21$, both heterozygous) in adenoma, 0.06 ( $2 / 33$, both heterozygous) in advanced adenoma, and 0.07 (4/56, all heterozygous) in CRCs (Table 2). One variant was mapped to the HMG box while 1 was to the activation domain (Figure 3E).

\section{TGFBR2 variants}

We found 169 variants in the discovery set, of which 157 were novel. From these, 142 were non-synonymous and 15 were stopgains. We validated 6 known variants (Table 2). Three were synonymous at locus Chr3: 30713674 exon 4 with codon change of CTA to CTG with a frequency of $0.20(6 / 30$, all heterozygous) in normal, $0.14(3 / 21$, all heterozygous) in adenoma (No Amino acid change), 0.09 (3/33, all heterozygous) in advanced adenoma, and 0.18 (10/56, all heterozygous) in CRC; variant at locus Chr 3: 30713842 exon 4 with codon change $C$ to $T$ with a frequency of 0.07 (2/30, both heterozygous) in normal, $0.05(1 / 21$, heterozygous) in adenoma, $0.06(2 / 33$, both heterozygous) in advanced adenoma, and 0.04 (2/56, both heterozygous) in CRC; variant at locus Chr: 30729931 exon 6 with codon change of GTC to GTT with a frequency of 0.07 (2/30, both heterozygous) in normal, and 0.04 (2/56, both heterozygous) in CRC; and 1 was non-synonymous at locus chr: 30713619 exon 4 with codon change of ACG to ATG with a frequency of $0.10(2 / 33$, both heterozygous $)$ in advanced adenoma (Table 2) (Amino acid change from $\mathrm{T}$ to M). The other 2 were flanking intronic. Four variants were mapped to the Cyt ProtKinase I, II and III domains, and 2 on the extracellular domain (Figure 3F).

\section{TP53 variants}

We found 162 variants in the discovery set, of which 51 were novel. From these, 49 were non-synonymous, 1 was stopgain, and 1 was frameshift variant. We validated 9 variants that were all known. There were 3 nonsynonymous variants at locus Chr17: 7579472 exon 3 with nucleotide change $\mathrm{G}$ to $\mathrm{C}$ and a frequency of 0.70 (21/30, 
all heterozygous) in normal, 0.67 (14/21, all heterozygous) in adenoma, 0.67 (22/33, all heterozygous) in advanced adenoma, and 0.61 (34/56, all heterozygous) in CRC (Amino acid change from $\mathrm{P}$ to $\mathrm{R}$ ); locus Chr17: 7578406 exon 1 with nucleotide change $\mathrm{C}$ to $\mathrm{T}$ with a frequency of $0.03(1 / 30$, heterozygous) in normal, and 0.05 (3/56, all heterozygous) in CRC (Amino acid change from R to $\mathrm{H}$ ); and locus Chr17: 7577120 exon 4 with nucleotide change $\mathrm{C}$ to $\mathrm{T}$ with a frequency of 0.04 (2/56, both heterozygous) in CRC (Amino acid change from $\mathrm{R}$ to $\mathrm{H}$ ). There was one synonymous variant at locus Chr17: 7578210 exon 2 with codon change of CGA to CGG with a frequency 0.05 (1/21, all heterozygous) in adenoma, and 0.02 (1/56, all heterozygous) in CRC. There were two stopgain variants at locus Chr17: 7578212 exon 2 with codon change GCT to ACT with a frequency of 0.07 (4/56, all heterozygous) in CRC (Amino acid change from $\mathrm{R}$ to $\mathrm{X}$ ); and locus Chr17: 7574003 exon 6 with nucleotide change $\mathrm{G}$ to A with a frequency 0.03 (1/33, all heterozygous) in advanced adenoma, and 0.02 (1/56, all heterozygous) in CRC (Amino acid change from $\mathrm{R}$ to $\mathrm{X})$. The remaining 3 variants were intronic (Table 2). We mapped 1 variant to the transactivation domain, 1 in the proline rich domain, 5 in the DNA binding domain, and 2 in the oligomerization domain (Figure 3G).

\section{SMAD4 variants}

We found 196 variants in the discovery set, of which 159 were novel. From these, 153 were non-synonymous, 4 were stopgains, and 2 were frameshift variants. We validated six intronic variants of which 2 were novel (Table 2). Variant at locus chr8: 48584624 with a $\mathrm{T}$ to $\mathrm{C}$ change had a frequency 0.02 (1/56, heterozygous) in CRCs. Variant at locus chr8: 48575700 with a $\mathrm{T}$ to $\mathrm{C}$ change had a frequency $0.02(1 / 56$, heterozygous) in CRCs. Of the 6 validated variants, three were found in the linker region and 3 in the $\mathrm{MH} 2$ region (Figure 3H).

\section{Oncogenes}

\section{$K R A S$ variants}

We found 54 variants in the discovery set, of which 44 were novel. From these, 41 were non-synonymous and 3 were stopgains. All variants were in the RAS domain. We validated seven known variants, 5 of which were non-
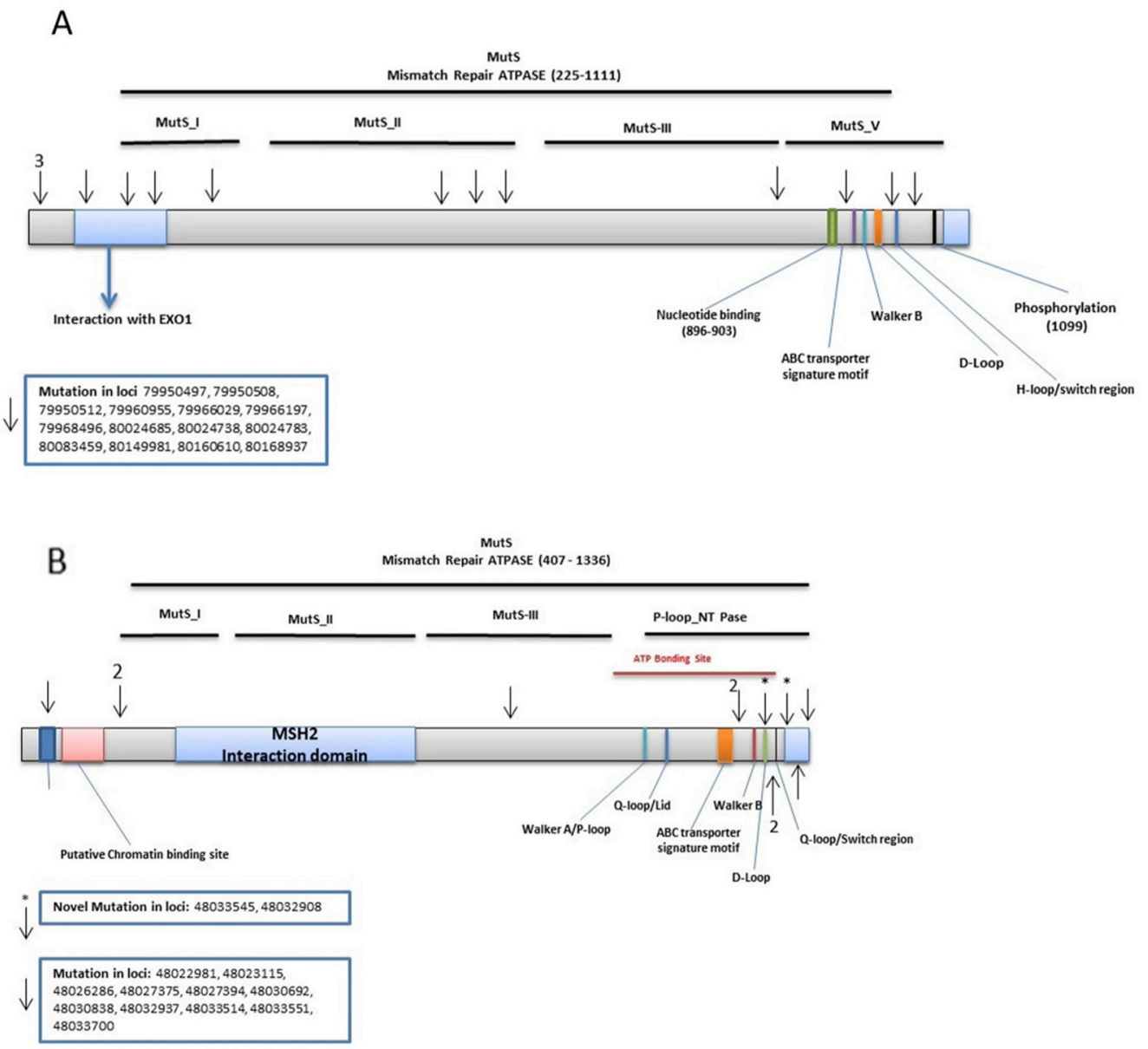

Figure 2: Distribution of validated variants per targeted MMR genes. (A) MSH3 (B) MSH6. 
synonymous, 1 synonymous, and 1 flanking intronic. The non-synonymous variants are as follows: locus 25398281 exon 2, a $\mathrm{C}$ to $\mathrm{T}$ change, with a frequency of $0.14(3 / 21$, all heterozygous) in adenoma, 0.15 (5/33, all heterozygous) in advanced adenoma, and $0.16(9 / 56$, all heterozygous) in CRC (Amino acid change from G to D); locus 25398284 exon 2 , a $\mathrm{C}$ to $\mathrm{T}$ change, with a frequency of 0.14 (3/21, all heterozygous) in adenoma, $0.06(2 / 33$, both heterozygous $)$ in advanced adenoma, and 0.21 (12/56, all heterozygous)
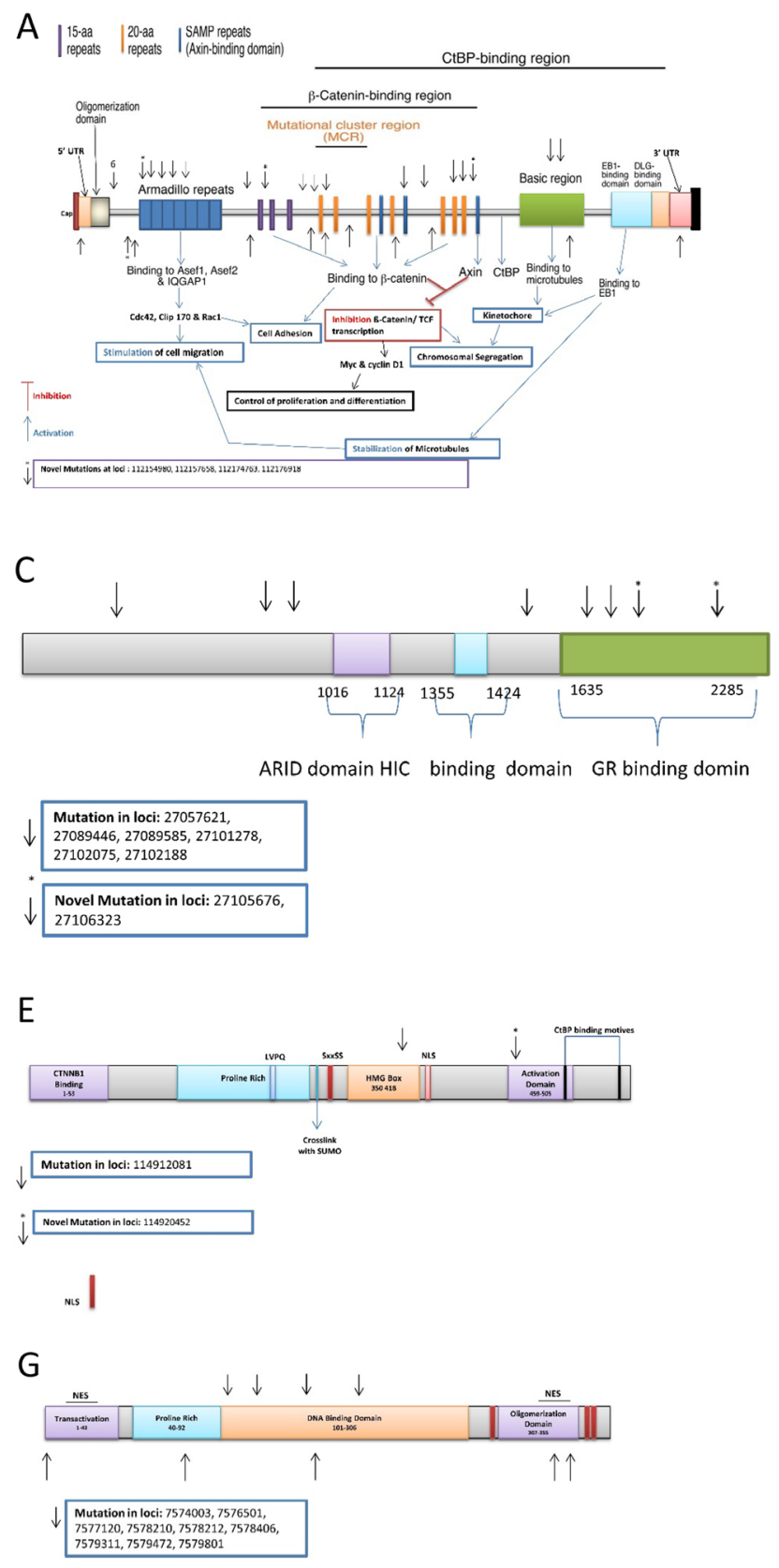

in CRC (Amino acid change from $\mathrm{G}$ to $\mathrm{D}$ ); locus 25398285 exon 2, a $\mathrm{C}$ to A change, with a frequency of $0.06(2 / 33$, both heterozygous) in advanced adenoma, and $0.02(1 / 56$, heterozygous) in CRC (Amino acid change from $\mathrm{G}$ to $\mathrm{C}$ ); locus 25398285 exon 2 , a $\mathrm{C}$ to $\mathrm{T}$ change, with a frequency of 0.09 (3/33, all heterozygous) in advanced adenoma, and 0.04 (2/56, both heterozygous) in CRC (Amino acid change from $\mathrm{G}$ to $\mathrm{S}$ ); locus 25398255 exon 2 with nucleotide change $\mathrm{G}$ to $\mathrm{T}$, and a frequency of 0.03 (1/33, heterozygous)
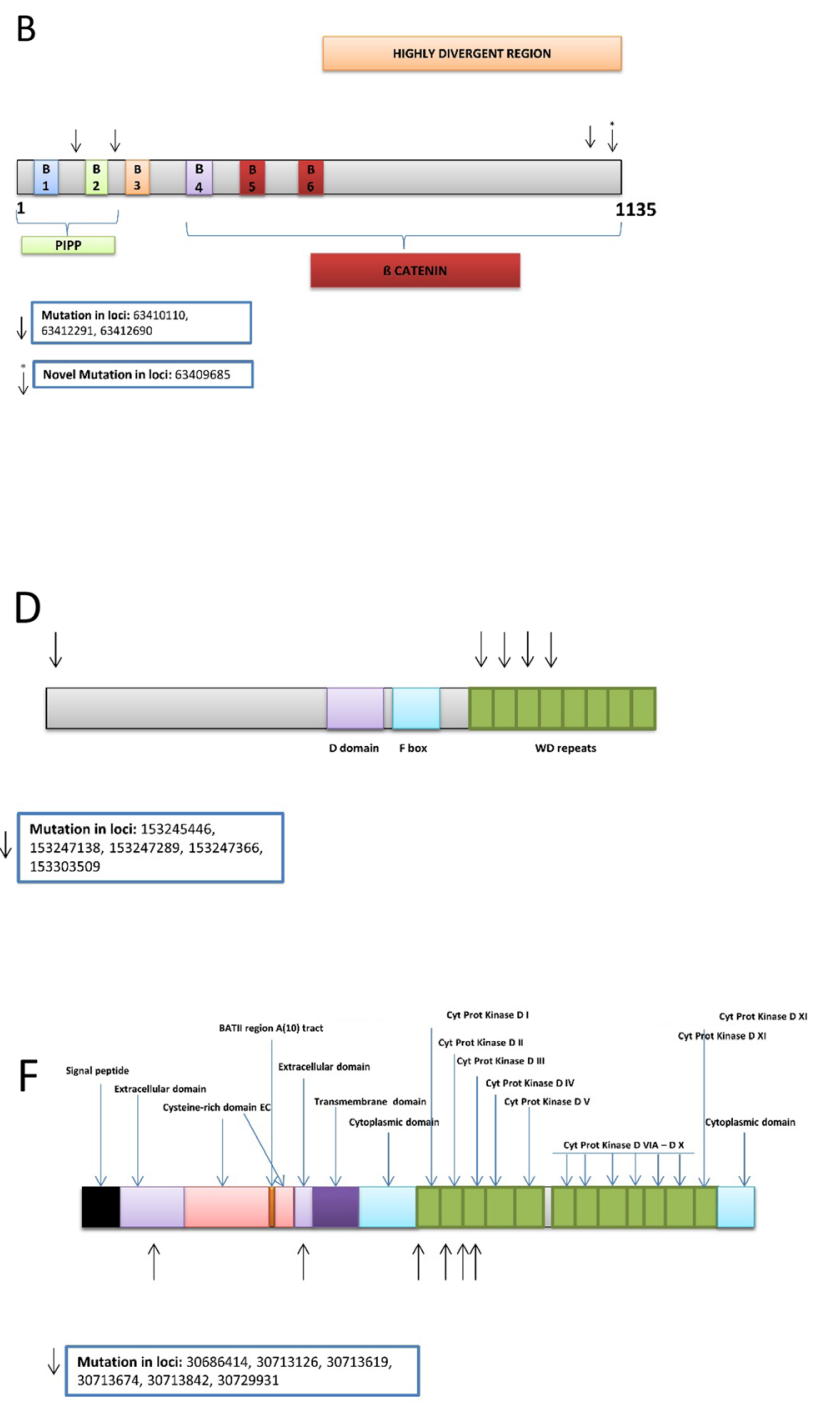

$\mathrm{H}$

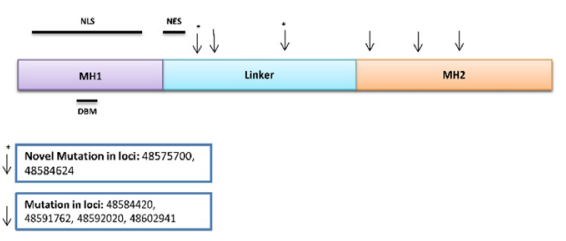

Figure 3: Tumor suppressor genes validated variants: (A) $A P C$, (B) $A M E R 1$, (C) $A R I D 1$, (D) FBXW7, (E) TCF7L2, (F) TGFBR2, (G) TP53, (H) SMAD4. 
in advanced adenoma (Table 2). Three variants were mapped in the GTP binding region, $1 \mathrm{C}$-terminal to the GTP binding region, (Amino acid change from $\mathrm{Q}$ to $\mathrm{K}$ ) and 3 in the hypervariable region (Figure 4A).

\section{$N R A S$ variants}

We found 57 variants in the discovery set, of which 50 were novel. From these, 44 were non-synonymous, 2 frameshift substitutions, and 4 stopgains. No NRAS variants were validated on the Illumina platform.

\section{$B R A F$ variants}

We found 221 variants in the discovery set, of which 200 were novel. From these, 184 were non-synonymous and 16 were stopgain variants. We validated 5 variants of which 1 was novel. One variant was non-synonymous, 1 synonymous, and 3 flanking intronic. Non-synonymous novel variant Chr7: 140487360 exon 9 has a frequency of 0.02 (1/56, heterozygous) in CRC. Synonymous variant Chr7: 140449150 exon 16 has a frequency of 0.77 (23/30, 13 homozygous and 10 heterozygous) in normal, 0.76 (16/21, 5 homozygous and 11 heterozygous) in adenoma, 0.85 (28/33, 8 homozygous and 20 heterozygous) in advanced adenoma, and 0.86 (48/56, 18 homozygous and 30 heterozygous) in CRC (Table 2). The variants were mapped prior and after CR3 (Figure 4B).

\section{PIK3CA variants}

We found 251 variants in the discovery set, of which 214 were novel. From these, 191 are non-synonymous and 23 were stopgain variants. We validated seven variants of which 1 was novel (Table 2). One variant was nonsynonymous while the other 6 were flanking intronic. Non-

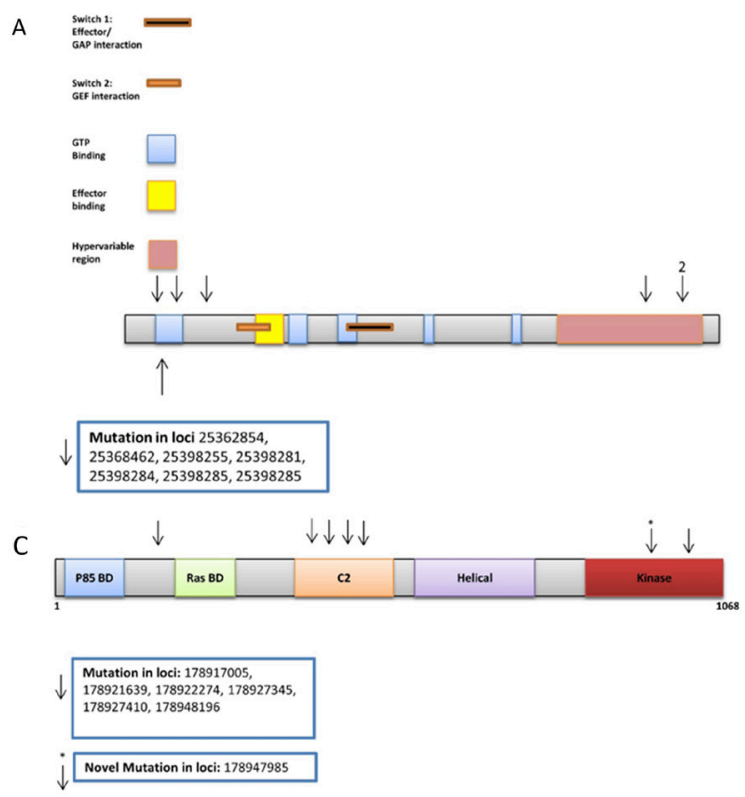

synonymous variant at locus Chr3: 178927410 exon 7, an A to $\mathrm{G}$ change, had a frequency of 0.27 (6/30, 2 homozygous and 4 heterozygous) in normal, (7/21, all heterozygous), 0.33 in adenoma, 0.42 (14/33, 2 homozygous and 12 heterozygous) in advanced adenoma and 0.41 (23/56, 1 homozygous and 22 heterozygous) in CRC. One variant was mapped prior to Ras $\mathrm{BD}$ region, 4 in the $\mathrm{C} 2$ domain, and 2 in the kinase domain (Table 2 and Figure 4C).

\section{SOX9 variants}

We found 189 variants in the discovery set, of which 183 were novel. From these, 162 were non-synonymous, 13 were stopgains, and 8 were frameshift variants. We validated three known variants; 1 synonymous and 2 flanking intronic (Table 2). Synonymous variant at locus Chr17: 70118935 had a frequency of 0.33 (10/30, all heterozygous) in normal, 0.14 (3/21, all heterozygous) in adenoma, 0.09 (3/33, all heterozygous) in advanced adenoma and 0.32 (17/56, 1 homozygous and 16 heterozygous) in CRCs (Table 2). One variant was mapped prior to the DIM region, 1 in the HMG region, and 1 in the TA region (Figure 4D).

\section{MMR genes}

MSH3: We found $315 \mathrm{MSH} 3$ variants in the discovery set, of which 298 were novel. We validated 14 known variants. Five of these variants were non-synonymous and altered exons 10, 21, and 23. Two were synonymous with changes in exon 4 and 18. Variant at locus 79966029 with a $\mathrm{G}$ to A change, with a frequency of $0.23(7 / 30,1$ homozygous and 6 heterozygous) in normal, 0.52 (11/21, all heterozygous) in adenoma, 0.21 ( $7 / 33,2$ homozygous and 5 heterozygous) in advanced adenoma, and $0.29(16 / 56$, all

$B$
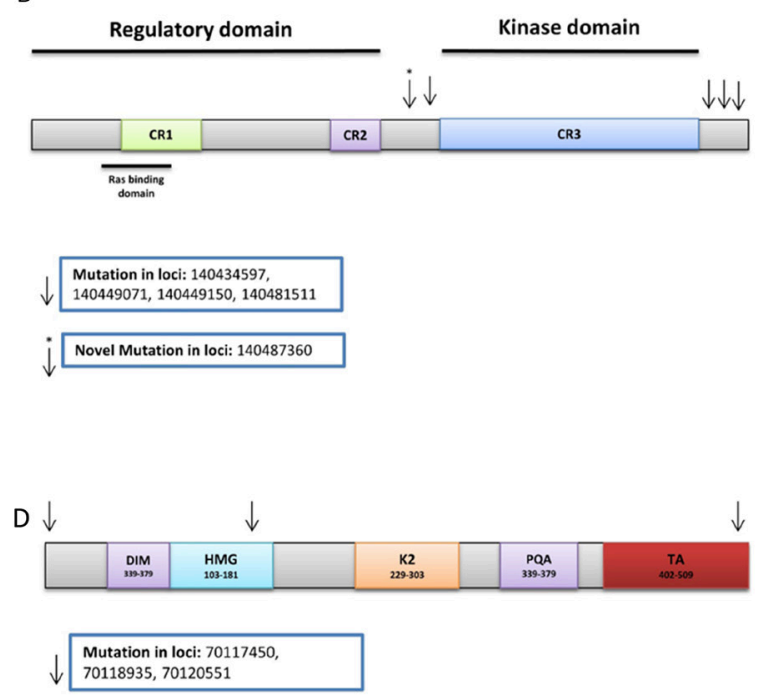

Figure 4: Oncogenes' validated variants: (A) KRAS, (B) BRAF, (C) PIK3CA, (D) SOX9. 
heterozygous) in CRCs. Variant at locus 80083459 with a $\mathrm{G}$ to A change, with a frequency of 0.03 (1/30, heterozygous) in normal, $0.06(2 / 33$, both heterozygous) in advanced adenoma, and 0.02 (1/56, heterozygous) in CRCs. The rest were flanking intronic (Table 2). The variants were mapped to the MSH3-MSH2-MSH6 region with 3 variants prior to EXO1, 3 in EXO1, 1 in MutS_I, 3 in MutS_II, 1 in MutS III, and 3 in MutS_V [5] (Figure 2A).

MSH6: We detected 434 variants in the discovery set, of which 396 were novel. The Illumina sequencing led to the validation of 12 variants. Two of these were novel. The $\mathrm{A}>\mathrm{G}$ and $\mathrm{A}>\mathrm{C}$ variants are flanking the region coding for the MutS-V domain and the MSH2 binding site, respectively. An $\mathrm{A}>\mathrm{C}$ intronic variant (IVS8-45) at locus chr2:48033545 was observed in $1 \mathrm{CRC}$ sample with a frequency of $0.02(1 / 56$, heterozygous). The $\mathrm{A}>\mathrm{G}$ variant at locus chr2:48032908 was intronic (IVS7-50) and was observed in 1 sample with a frequency of $0.03(1 / 30$, heterozygous) in normal, 0.05 $(1 / 21$, heterozygous) in adenoma, and 0.04 (2/56, both heterozygous) in CRC (Table 2). One variant was mapped in PWWP, 2 prior to the MutS_I, 1 in MutS_III, and 8 in P-loop_NTPase [5] (Figure 2B).

\section{Summary of validated variants}

In total, there were 12 validated variants in the 14 genes: APC (33/121 [27\%]), AMER1 (4/121) [3\%]), ARID1 (8/121 [7\%]), MSH3 (14/121 [12\%]), MSH6 (12/121 [10\%]), BRAF (5/121 [4\%]), KRAS (7/121 [6\%]), FBXW7 (5/121 [4\%]), PIK3CA (7/121 [6\%]), SMAD4 (6/121 [5\%]), SOX9 (3/121 [2\%]), TCF7L2 (2/121 [2\%]), TGFBR2 (6/121 [5\%]), TP53 (9/121 [7\%]). From these validated variants, $14 / 121(12 \%)$ were novel variants in 8 genes (AMER1, APC, ARID1A, BRAF, MSH6, PIK3CA, $S M A D 4$, and TCF7L2). Of the validated variants, $23 \%$ (28/121) were non-synonymous, 14\% (17/121) were stopgains, 24\% (29/121) were synonymous and 39\% (47/121) were intronic variants.

\section{DISCUSSION}

We performed a targeted exome sequencing in African Americans with colorectal lesions with the goal of detecting particular variant profiles that can partially explain the observed CRC disparity in this population through the identification of highly pathogenic and frequent variants and mutations. While most genes displayed a high number of variants (novel and known) on one platform (Ion Torrent), not all were validated on the second sequencing platform (HiSeq, Illumina). We previously reported such discrepancies on our recent study [33] thus mandating a necessary validation step that we performed on the HiSeq platform. We validated 121 variants in 14 mechanistically known driver genes including Tumor Suppressor Genes (TSGs): APC (33/121 [27\%]), AMER1 (4/121) [3\%]), ARID1 (8/121 [7\%]),
FBXW7 (5/121 [4\%]), SMAD4 (6/121 [5\%]), TCF7L2 (2/121 [2\%]), TGFBR2 (6/121 [5\%]), TP53 (9/121 [7\%]), oncogenes: PIK3CA (7/121 [6\%]), BRAF (5/121 [4\%]), $\operatorname{KRAS}(7 / 121[6 \%]), S O X 9$ (3/121 [2\%]), and MMR genes, MSH3 (14/121 [12\%]), MSH6 (12/121 [10\%]). From these validated variants, $14 / 121(12 \%)$ were novel variants in 8 genes (AMER1, APC, ARID1A, BRAF, MSH6, PIK3CA, $S M A D 4$, and TCF7L2). Of the validated variants, $23 \%$ $(28 / 121)$ were non-synonymous, $14 \%$ (17/121) were stopgains, 24\% (29/121) were synonymous and 39\% (47/121) were intronic variants. A sizable portion of the validated variants $(39 \%)$ were intronic. While it is difficult to assess the potential effects of such variants on the protein product and function, many studies have shown that such variants lead to aberrant splicing with different levels of impact on the protein function in many pathological diseases [34].

Here, we report the profile of variants in African American colorectal lesions using targeted exome sequencing. Validated variants showed that the tumor suppressor genes $A P C$ and $A R I D 1$ and the DNA MMR genes MSH3 and MSH6 are the genes with the highest numbers of validated variants. Oncogenes KRAS and $P I K 3 C A$ were also primary variant targets that likely participated in increased proliferative potential of the mutated colonic epithelial cells in this population. Many of these recurrent and frequent variants were novel and not previously reported in any of the known databases.

The 8 TSGs (AMER1, APC, ARID, FBXW7, TCF7L2, $T G F B R 2$, SMAD4, and TP53) in our gene panel accounted for $59 \%$ of the validated variants. If added to the $20 \%$ of validated DNA MMR genes' variants, this will give a 73\% rate of validated variants within TSGs. This finding is in line with the importance of TSGs in the initiation of the carcinogenic process and with the fact that such genes need double-hits for complete inactivation, unlike oncogenes. More than 2/3 TSGs validated variants in our study reflects perfectly the roles and rates at which such genes are targeted in the neoplastic transformation process.

$A P C$ is part of WNT pathway in CRC and was the top target gene with $27 \%(33 / 121)$ of the validated variants (4 novel variants, Table 3). Its role for downstream signaling with B-catenin, GSK and AXIN has been well established in previous studies [35]. Wnt/ $\beta$-catenin pathway plays multiple and diverse roles in development by regulating gene expression via T-cell factor/Lymphoid enhancer-binding factor (Tcf/Lef) DNA binding factors [36]. Angus-Hill et al. showed that Tcf4 (Tcf7L2) functions as a tumor suppressor gene in colon carcinogenesis [36].

Exome Sequencing has revealed $A M E R 1$ as a frequent target in CRC [37]. In our study, we report one novel variant with a frequency of 0.07 in normal, 0.05 in adenoma, 0.03 in advanced adenoma and 0.02 in CRC samples. Overexpression of AMER1 increases the expression of APC and causes subcellular re-localization of APC from the microtubule ends to the plasma membrane of epithelial cells [37]. As such, 
Table 3: Distribution of validated variants in signaling pathways

\begin{tabular}{|c|c|c|c|c|c|c|}
\hline Gene & $\begin{array}{l}\text { Signaling } \\
\text { Pathway }\end{array}$ & $\begin{array}{c}\text { TSG } \\
\text { vs. Oncogene }\end{array}$ & Function & $\begin{array}{c}\text { Total \# } \\
\text { of Variants } \\
(n=121)\end{array}$ & $\begin{array}{c}\text { Total } \\
\text { Variant } \\
\text { FRQ }\end{array}$ & $\begin{array}{c}\text { Novel } \\
\text { Variant } \\
\text { n, } \%\end{array}$ \\
\hline AMER1 & Wnt $/ \beta$-catenin & TSG & $\begin{array}{l}\text { Proliferation, subcellular } \\
\text { distribution of APC }\end{array}$ & 4 & $3 \%$ & 1,1 \\
\hline APC & Wnt $/ \beta$-catenin & TSG & Proliferation & 33 & $27 \%$ & 4,3 \\
\hline ARID1A & Wnt $/ \beta$-catenin & TSG & Proliferation & 8 & $7 \%$ & 2,2 \\
\hline BRAF & RTK-RAS & Oncogene & $\begin{array}{c}\text { Cell } \\
\text { survival, translation, } \\
\text { proliferation }\end{array}$ & 5 & $4 \%$ & 1,1 \\
\hline FBXW7 & Wnt $/ \beta$-catenin & TSG & Proliferation & 5 & $4 \%$ & No \\
\hline KRAS & RTK-RAS & Oncogene & $\begin{array}{c}\text { Cell } \\
\text { survival, translation, } \\
\text { proliferation }\end{array}$ & 7 & $6 \%$ & No \\
\hline MSH3 & Mismatch repair & $\begin{array}{l}\text { Suppression of } \\
\text { Tumor }\end{array}$ & Mismatch repair & 14 & $12 \%$ & No \\
\hline MSH6 & Mismatch repair & $\begin{array}{l}\text { Suppression of } \\
\text { Tumor }\end{array}$ & Mismatch repair & 13 & $11 \%$ & 2,2 \\
\hline $\begin{array}{l}\text { MMR (MSH3*, } \\
\text { MSH6, MSH2, } \\
\text { PMS2, MLH1 }\end{array}$ & Mismatch repair & $\begin{array}{l}\text { Suppression of } \\
\text { Tumor }\end{array}$ & Mismatch repair & 27 & $23 \%$ & 2,2 \\
\hline PIK3CA & PI3K-Akt & Oncogene & $\begin{array}{c}\text { Cell survival, translation, } \\
\text { proliferation }\end{array}$ & 7 & $6 \%$ & 1,1 \\
\hline SMAD4 & Wnt $/ \beta$-catenin & Oncogene & Proliferation & 6 & $5 \%$ & 2,2 \\
\hline SOX9 & Wnt $/ \beta$-catenin & Oncogene & $\begin{array}{c}\text { Proliferation, } \\
\text { Self-Renewal } \\
\text { of } \\
\text { Oncogene } \\
\text { Targeted Cells }\end{array}$ & 3 & $2 \%$ & No \\
\hline TCF7L2 & Wnt $/ \beta$-catenin & $\begin{array}{l}\text { Both (TSG/ } \\
\text { Oncogene) }\end{array}$ & Proliferation & 2 & $2 \%$ & 1,1 \\
\hline$\beta R 2$ & $\begin{array}{l}\text { TGF- } \beta \text { (growth } \\
\text { factor) }\end{array}$ & TSG & Proliferation & 6 & $5 \%$ & No \\
\hline TP53 & P53 & TSG & $\begin{array}{l}\text { Proliferation, } \\
\text { Cell survival }\end{array}$ & 9 & $7 \%$ & No \\
\hline
\end{tabular}

variants within this gene may downregulate the APC gene and downstream signaling pathways. SMAD4 is a well-established tumor suppressor gene that displayed a high number of novel variants $(\mathrm{n}=112)$, however none were validated on the Illumina platform. Only 4 known variants were validated and they were all mapped to the linker region (Figure $3 \mathrm{H}$ ).

ARIDIA has been identified as a novel tumor suppressor gene in ovarian cancer and subsequently in various other tumor types. ARIDIA encodes a protein that belongs to the ARID domain containing family, which consists of 15 genes encoding proteins involved in transcriptional regulation, proliferation and chromatin remodeling [38]. There were 7\% (8/121) validated variants highlighting a potential bigger role of this TSG in CRC in African Americans (Table 2, Figure 3C).

TGFBR2 signaling is involved in cell-cell communication, cell adhesion and cell migration. The role of this pathway in the glycosylation pattern of cell surface proteins is largely unexplored. Experimental evidence suggested a possible link between mutated MSI target genes and the glycosylation pattern at the cell surface [39]. We detected 5\% (6/121) of the variants in TGFBR2 gene (Table 3), 1 non-synonymous variant at locus Chr: 30713619 exon 4 with codon change of ACG to ATG that mapped to the Cyt ProtKinase I, II and III domain (Figure 3F). This variant could have an effect on tumor invasive phenotype. 
$F B X W 7$ has been identified as a transcriptional target of TP53 and lower expression levels of $F B X W 7$ in correlation with TP53-variants have been reported [40]. $F B X W 7$ loss leads to induction of p53-phosphorylation at Serine-15, p53 stabilizes in the nucleus to act as a transcriptional activator for tumor suppression, implicating phospho-p53 (Ser15) as a marker of $F B X W 7$-associated carcinogenesis [27]. We found intronic and nonsynonymous variants both in advanced adenoma and in CRCs (Table 2). All four variants were mapped to the WD repeat domain of the protein (Figure 3D). The mutations in this gene may contribute to the decreased efficacy of therapy in FBXW7-mutated CRC [27].

TP53 is frequently inactivated by variant or deletion in most human tumors [41]. A tremendous effort has been made to restore TP53 activity in cancer therapies. However, no effective p53-based therapy has been successfully translated into clinical cancer treatment owing to the complexity of p53 signaling [41]. For the TP53 gene, there were $7 \%(9 / 121)$ validated that were mapped (Figure $3 \mathrm{G}$ ). The potential deleterious effect of these variants needs experimental validation.

The oncogenes within the sequenced gene panel had a total of 22 out of 121 validated variants (PIK3CA (7/121 [6\%]), BRAF (5/121 [4\%]), KRAS (7/121 [6\%]), SOX9 $(3 / 121[2 \%]))$. We previously reported on variants targeting $B R A F$ and $K R A S$ in African Americans with colorectal cancer, however the present data seems to give weight to other oncogenes such as $P I K 3 C A$ as major targets of variants.

Establishing $K R A S$ variants' status in each patient is indeed important to determine the appropriate therapy, patients with wild-type $K R A S$ could receive monoclonal antibodies against EGFR [42] while KRAS mutated patients have been associated with no-response to targeted therapies and poor prognosis in different studies [43]. In our study, we found 1 non-synonymous known variant with a 0.03 frequency in patients with advanced adenoma (Table 2). There were no detected variants in the notable hotspots of $K R A S$ (codons 12/13/61/144) [44].

Variants in the $B R A F$ oncogene is a key step in malignant transformation within a subset of CRCs, generally MSI-H and with the CpG methylator phenotype [16]. It is unclear to what extent the lack of response in $K R A S$ wild-type CRCs is due to $B R A F$ variants, but data suggest that mutated $B R A F$ confers resistance to anti-EGFR therapy $[45,46]$. According to Palmirotta et al., a $K R A S$ variant $(\mathrm{CAG}>\mathrm{TAG})$ determining a premature stop signal at codon 22 (Gln22Stop) has been previously found in a patient with metastatic colorectal cancer [47]. Whether or not the validated stopgain variants in $K R A S$ associate with an activating effect remains to be explored and investigated.

The prevalence of $P I K 3 C A$ variants increases continuously from rectal to cecum cancers, supporting the 'colorectal continuum' paradigm, and an important interplay of gut microbiota and host immune/inflammatory reaction [48]. PIK3CA variants also contribute to significantly decreased survival for patients with wildtype $B R A F$ tumors [49]. Numerous studies have shown that variants are concentrated in 2 hotspots of the $P I K 3 C A$ gene: the helical domain in codons 542 and 545 of exon 9 and the kinase domain in codon 1047 of exon 20 [49]. In our study, $P I K 3 C A$ variants were $6 \%$ of the validated variants (Table 2). The novel variant was intronic with a CRC frequency of 0.02 (Table 2). This variant was mapped to N-terminus region before the Kinase region of the PIK3CA. The known variant was also intronic with a variants' frequency of 0.2 in normal, 0.29 in adenoma, 0.12 in advanced adenoma, and 0.11 in CRCs (Table 2). Recently Kim et al, showed that $P I K 3 C B$ rare point variant is associated with tumorigenesis [50]. This somatic variant event in the cancer, supports our results that low frequency of the variants may have tumorigenic function that needs verification with in vivo and in vitro analysis.

In the intestinal epithelium, $S O X 9$ is expressed in the stem/progenitor cells, as well as in the nuclei of terminally differentiated Paneth cells of the small intestinal crypts and tuft cells in the villi. It plays a crucial role in Paneth cell differentiation $[19,51]$. From our panel, 2\% (3/124) of validated variants were in this gene. This corresponds to an intronic variant with a frequency of 0.1 in normal, 0.05 in adenoma, 0.06 in advanced adenoma and 0.09 in CRC samples (Table 2). This variant was mapped to the DIM region of SOX9 (Figure 4D).

Among MMR genes, 26 of the validated variants were in MSH3 and MSH6 genes accounting for $22 \%$ of the validated variants [5]. This finding highlights the important role that these genes play in the setting of genome-wide instability aside from the already well described roles of $M L H 1$ and $M S H 2$ genes in this process. Indeed, variants within these genes, and specifically within $M S H 3$, have been linked to the EMAST phenotype characterized by instability within tetranucleotide repeats. Patients with such phenotype have a poor prognosis. This might help partly understand colorectal cancer disparity in African Americans. Indeed, EMAST is reported to be highly prevalent (50 to $60 \%$ ) in this population when compared to others [11].

The lack of matching normal for many of the analyzed the samples, and the absence of MSH2, POLE, MLH1 and $S M A D 2$ on our targeted exome panel constitute limitations to our study. However, our study is a good example for other more comprehensive studies that will use larger sample size and more inclusive gene panels particularly for African American patients which is under-represented.

In this study, we examined and validated variants in 15 driver cancer genes in African Americans with colorectal neoplastic lesions. Many of these driver genes are involved and different pathways are affected by these variants which are all part of the carcinogenic process. Among oncogenes and TSGs, PIK $3 C A$ and $A P C$ genes were the most frequently altered genes, respectively. DNA MMR genes MSH3 and MSH6 also displayed a high level 
of variants that probably affect overall genetic stability. The distinct variant profiles with novel variants can help to predict, diagnose and establish new targeted therapeutic modalities for optimal CRC patients' care.

\section{MATERIALS AND METHODS}

\section{Discovery set}

A total of 140 colorectal samples including 30 normals, 21 adenomas, 33 advanced adenomas $(>1 \mathrm{~cm}$ and/or with villous histology), and 56 cancers were used to establish variant profiles by targeted exome sequencing, using a PGM sequencing platform (Figure 1). These samples were collected from 123 patients at different stages of the disease. The Howard University Institutional board approved (06-MED-39) the collection of archival unidentified human samples.

\section{Validation set}

A total of 36 samples from 26 patients including 12 normals, 5 adenomas, 4 advanced adenomas and 15 cancers, were used to validate the Ion Torrent detected variants on a second sequencing platform (HiSeq, Illumina). The validation set is a subset from the discovery set samples (Figure 1).

\section{Proton (Ion Torrent) NGS}

A targeted, multiplex PCR panel was designed using the custom Ion AmpliSeq Designer v1.2 (ThermoFisher Scientific, Grand Island, NY). The panel amplified $56.9 \mathrm{~kb}$ and included the coding regions of 20 genes, with an average coverage of $96.9 \%$ of the protein coding regions and splice junctions ( +5 intronic bases). In this study, we only report data from the 15 genes that are common to the Illumina gene panel that were sequenced in the validation set. The panel was designed to amplify PCR products with an average amplicon size of 150 base pairs (bp). Sample DNA (20 ng/primer pool) was amplified using the PCR panel, and libraries were prepared using the AmpliSeq Library Preparation kit following the manufacturer's protocol (Thermo-Fisher Scientific, Grand Island, NY). Individual samples were barcoded, pooled, and sequenced on a Proton Sequencer using the Ion PI Template OT2 200v3 and Ion PI Sequencing 200v2 kits per the manufacturer's instructions. Raw sequencing reads were filtered for high quality reads and the adaptors were removed using the Ion Torrent Suite 4.0.4, then reads were aligned to the hg19 reference sequence by TMAP (https://github.com/iontorrent/TS/ tree/master/Analysis/TMAP) using default parameters. Resulting BAM files were processed through an in-house quality control (QC) filter and coverage analysis pipeline. BAM files were aligned using the GATK LeftAlignIndels module. Amplicon primers were trimmed from aligned reads using the Torrent Suite. Variant calls were made by the Torrent Variant Caller 4.0 (https://www.edgebio.com/ variant-calling-ion-torrent-data).

\section{HiSeq (Illumina) NGS}

DNA quantification and quality assessment for the validation set, NGS using a HiSeq platform (Illumina, San Diego, CA), SNV and indel detection, and assessment of copy number alterations were performed as previously described $[5,6]$.

\section{Bioinformatics and comparison of African Americans' variants to available databases}

We used $\mathrm{R}$ software (version 2.15.2, http://www.r-project.org) to compare the variants in the normal and tumor samples with publicly available databases. Variants were annotated using ANNOVAR (56) and the 1000Genomes database, which represents a nominally noncancerous population. TCGA and GENIE databases were used to compute the frequency of mutations in another colorectal cancer database (TCGA) and a nominally noncancerous population (GENIE). All samples displayed more or less an equal number of SNVs in their tumors compared with their matched normal samples. We would like to make it clear that variants identified using both platforms allowed us to identify weaknesses in bioinformatics associated with each platform that were addressed by rigorous manual review that improved variant detection on each individual platform. Subsequent to incorporating the more rigorous review, we identified additional variants with high confidence using a single platform.

\section{Author contributions}

Conception and design: HA, HB

Development of methodology: MN, SV, HAz

Acquisition of data (acquired and managed patients and samples): HAz, EL, AOL,

Analysis and interpretation of data (e.g., statistical analysis, biostatistics, computational analysis): SV, HA, $\mathrm{HB}, \mathrm{MN}$ $\mathrm{HA}, \mathrm{HB}$

Writing, review, and/or revision of the manuscript:

Administrative, technical, or material support (i.e., reporting, organizing data): $\mathrm{HA}, \mathrm{HB}$

Study supervision: HA

\section{CONFLICTS OF INTEREST}

No potential conflicts of interest were disclosed.

\section{GRANT SUPPORT}

This project was supported (in part) by the National Institute on Minority Health and Health Disparities of 
the National Institutes of Health under Award Number G12MD007597.

\section{REFERENCES}

1. DeSantis CE, Siegel RL, Sauer AG, Miller KD, Fedewa SA, Alcaraz KI, Jemal A. Cancer statistics for African Americans, 2016: progress and opportunities in reducing racial disparities. CA Cancer J Clin. 2016; 66:290-308. https://doi.org/10.3322/caac.21340.

2. Carethers JM. Racial and ethnic factors in the genetic pathogenesis of colorectal cancer. J Assoc Acad Minor Phys. 1999; 10:59-67.

3. Satia JA, Keku T, Galanko JA, Martin C, Doctolero RT, Tajima A, Sandler RS, Carethers JM. Diet, lifestyle, and genomic instability in the North Carolina Colon Cancer Study. Cancer Epidemiol Biomarkers Prev. 2005; 14:429-36.

4. Guda K, Veigl ML, Varadan V, Nosrati A, Ravi L, Lutterbaugh J, Beard L, Willson JK, Sedwick WD, Wang ZJ, Molyneaux N, Miron A, Adams MD, et al. Novel recurrently mutated genes in African American colon cancers. Proceedings of the National Academy of Sciences of the United States of America. 2015; 112:1149-54. https://doi.org/10.1073/pnas.1417064112.

5. Ashktorab H, Azimi H, Varma S, Tavakoli P, Nickerson ML, Brim H. Distinctive DNA mismatch repair and APC rare variants in African Americans with colorectal neoplasia. Oncotarget. 2017; 8:99966-77. https://doi.org/10.18632/oncotarget.21557.

6. Ashktorab H, Daremipouran M, Devaney J, Varma S, Rahi H, Lee E, Shokrani B, Schwartz R, Nickerson ML, Brim H. Identification of novel mutations by exome sequencing in African American colorectal cancer patients. Cancer. 2015; 121:34-42. https://doi.org/10.1002/cncr.28922.

7. Ashktorab H, Ahuja S, Kannan L, Llor X, Nathan E, Xicola RM, Adeyinka LO, Carethers JM, Brim H, Nouraie M. A meta-analysis of MSI frequency and race in colorectal cancer. Oncotarget. 2016; 7:34546-57. https://doi.org/10.18632/oncotarget.8945.

8. Ashktorab H, Daremipouran M, Goel A, Varma S, Leavitt $\mathrm{R}$, Sun X, Brim H. DNA methylome profiling identifies novel methylated genes in African American patients with colorectal neoplasia. Epigenetics. 2014; 9:503-12. https://doi.org/10.4161/epi.27644.

9. Ashktorab H, Rahi H, Wansley D, Varma S, Shokrani B, Lee E, Daremipouran M, Laiyemo A, Goel A, Carethers JM, Brim H. Toward a comprehensive and systematic methylome signature in colorectal cancers. Epigenetics. 2013; 8:807-15.

10. Haugen AC, Goel A, Yamada K, Marra G, Nguyen TP, Nagasaka T, Kanazawa S, Koike J, Kikuchi Y, Zhong X, Arita M, Shibuya K, Oshimura M, et al. Genetic instability caused by loss of MutS homologue 3 in human colorectal cancer. Cancer Res. 2008; 68:8465-72. https://doi.org/10.1158/0008-5472.CAN-08-0002.

11. Carethers JM, Koi M, Tseng-Rogenski SS. EMAST is a form of microsatellite instability that is initiated by inflammation and modulates colorectal cancer progression. Genes (Basel). 2015; 6:185-205. https://doi.org/10.3390/genes6020185.

12. Venderbosch $\mathrm{S}$, van Lent-van Vliet $\mathrm{S}$, de Haan AF, Ligtenberg MJ, Goossens M, Punt CJ, Koopman M, Nagtegaal ID. EMAST is associated with a poor prognosis in microsatellite instable metastatic colorectal cancer. PLoS One. 2015; 10:e0124538. https://doi.org/10.1371/journal.pone.0124538.

13. Zocche DM, Ramirez C, Fontao FM, Costa LD, Redal MA. Global impact of KRAS mutation patterns in FOLFOX treated metastatic colorectal cancer. Front Genet. 2015; 6:116. https://doi.org/10.3389/fgene.2015.00116.

14. Barresi V, Bonetti LR, Bettelli S. KRAS, NRAS, BRAF mutations and high counts of poorly differentiated clusters of neoplastic cells in colorectal cancer: observational analysis of 175 cases. Pathology. 2015; 47:551-6. https://doi.org/10.1097/PAT.0000000000000300.

15. Vandrovcova J, Lagerstedt-Robinsson K, Pahlman L, Lindblom A. Somatic BRAF-V600E mutations in familial colorectal cancer. Cancer Epidemiol Biomarkers Prev. 2006; 15:2270-3. https://doi.org/10.1158/1055-9965.EPI-06-0359.

16. Kalady MF, Dejulius KL, Sanchez JA, Jarrar A, Liu X, Manilich E, Skacel M, Church JM. BRAF mutations in colorectal cancer are associated with distinct clinical characteristics and worse prognosis. Dis Colon Rectum. 2012; 55:128-33. https://doi.org/10.1097/DCR.0b013e31823c08b3.

17. Miled N, Yan Y, Hon WC, Perisic O, Zvelebil M, Inbar Y, Schneidman-Duhovny D, Wolfson HJ, Backer JM, Williams RL. Mechanism of two classes of cancer mutations in the phosphoinositide 3-kinase catalytic subunit. Science. 2007; 317:239-42. https://doi.org/10.1126/science.1135394.

18. Muzny DM, Bainbridge MN, Chang K, Dinh HH, Drummond JA, Fowler G, Kovar CL, Lewis LR, Morgan MB, Newsham IF, Reid JG, Santibanez J, Shinbrot E, et al. Comprehensive molecular characterization of human colon and rectal cancer. Nature. 2012; 487:330-7. https://doi.org/10.1038/Nature11252.

19. Shi Z, Chiang CI, Labhart P, Zhao Y, Yang J, Mistretta TA, Henning SJ, Maity SN, Mori-Akiyama Y. Contextspecific role of SOX9 in NF-Y mediated gene regulation in colorectal cancer cells. Nucleic Acids Res. 2015; 43:625769. https://doi.org/10.1093/nar/gkv568.

20. Segditsas S, Rowan AJ, Howarth K, Jones A, Leedham S, Wright NA, Gorman P, Chambers W, Domingo E, Roylance RR, Sawyer EJ, Sieber OM, Tomlinson IP. APC and the three-hit hypothesis. Oncogene. 2009; 28:146-55. https://doi.org/10.1038/onc.2008.361.

21. Schneikert J, Brauburger K, Behrens J. APC mutations in colorectal tumours from FAP patients are selected for CtBPmediated oligomerization of truncated APC. Hum Mol Genet. 2011; 20:3554-64. https://doi.org/10.1093/hmg/ddr273. 
22. Thiagalingam S, Lengauer C, Leach FS, Schutte M, Hahn SA, Overhauser J, Willson JK, Markowitz S, Hamilton SR, Kern SE, Kinzler KW, Vogelstein B. Evaluation of candidate tumour suppressor genes on chromosome 18 in colorectal cancers. Nat Genet. 1996; 13:343-6. https://doi.org/10.1038/ng0796-343.

23. Shin YK, Yoo BC, Hong YS, Chang HJ, Jung KH, Jeong SY, Park JG. Upregulation of glycolytic enzymes in proteins secreted from human colon cancer cells with 5-fluorouracil resistance. Electrophoresis. 2009; 30:2182-92. https://doi.org/10.1002/elps.200800806.

24. Lee SY, Kim DW, Lee HS, Ihn MH, Oh HK, Park DJ, Kim HH, Kang SB. Loss of AT-rich interactive domain $1 \mathrm{~A}$ expression in gastrointestinal malignancies. Oncology. 2015; 88:234-40. https://doi.org/10.1159/000369140.

25. de Miranda NF, van Dinther M, van den Akker BE, van Wezel T, ten Dijke P, Morreau H. Transforming Growth Factor $\beta$ Signaling in Colorectal Cancer Cells With Microsatellite Instability Despite Biallelic Mutations in TGFBR2. Gastroenterology. 2015; 148:1427-37.e8. https://doi.org/10.1053/j.gastro.2015.02.052.

26. Papageorgis P. Complex interplay between aging and cancer: role of TGF-beta signaling. Crit Rev Oncog. 2017; 22:31321. https://doi.org/10.1615/CritRevOncog.2017025134.

27. Li N, Lorenzi F, Kalakouti E, Normatova M, BabaeiJadidi R, Tomlinson I, Nateri AS. FBXW7-mutated colorectal cancer cells exhibit aberrant expression of phosphorylated-p53 at Serine-15. Oncotarget. 2015; 6:924056. https://doi.org/10.18632/oncotarget.3284.

28. Markowitz SD, Bertagnolli MM. Molecular origins of cancer: molecular basis of colorectal cancer. N Engl J Med. 2009; 361:2449-60. https://doi.org/10.1056/NEJMra0804588.

29. Grohmann A, Tanneberger K, Alzner A, Schneikert J, Behrens J. AMER1 regulates the distribution of the tumor suppressor APC between microtubules and the plasma membrane. J Cell Sci. 2007; 120:3738-47. https://doi.org/10.1242/jcs.011320.

30. Mur P, Aiza G, Sanz-Pamplona R, Gonzalez S, Navarro M, Moreno V, Capella G, Valle L. AMER1 is a frequently mutated gene in colorectal cancer--letter. Clin Cancer Res. 2015; 21:4985. https://doi.org/10.1158/1078-0432.CCR-15-1756.

31. Folsom AR, Pankow JS, Peacock JM, Bielinski SJ, Heiss G, Boerwinkle E. Variation in TCF7L2 and increased risk of colon cancer: the atherosclerosis risk in communities (ARIC) study. Diabetes Care. 2008; 31:905-9. https://doi.org/10.2337/dc07-2131.

32. Shen J, Fang Y, Ge W. Polymorphism in the transcription factor 7-like 2 (TCF7L2) gene is associated with impaired proinsulin conversion--A meta-analysis. Diabetes Res Clin Pract. 2015; 109:117-23. https://doi.org/10.1016/j.diabres.2015.04.020.

33. Ashktorab H, Azimi H, Nickerson ML, Bass S, Varma $\mathrm{S}$, Brim H. Targeted Exome Sequencing Outcome variations of colorectal tumors within and across two sequencing platforms. Next Gener Seq Appl. 2016; 3:123. https://doi.org/10.4172/2469-9853.1000123.

34. Ward AJ, Cooper TA. The pathobiology of splicing. J Pathol. 2010; 220:152-63. https://doi.org/10.1002/path.2649.

35. Li L, Sheng Y, Li W, Hu C, Mittal N, Tohyama K, Seba A, Zhao YY, Ozer H, Zhu T, Qian Z. Betacatenin is a candidate therapeutic target for myeloid neoplasms with del(5q). Cancer Res. 2017; 77:4116-26. https://doi.org/10.1158/0008-5472.CAN-17-0202.

36. Angus-Hill ML, Elbert KM, Hidalgo J, Capecchi MR. T-cell factor 4 functions as a tumor suppressor whose disruption modulates colon cell proliferation and tumorigenesis. Proc Natl Acad Sci U S A. 2011; 108:4914-9. https://doi.org/10.1073/pnas.1102300108.

37. Sanz-Pamplona R, Lopez-Doriga A, Pare-Brunet L, Lazaro K, Bellido F, Alonso MH, Ausso S, Guino E, Beltran S, Castro-Giner F, Gut M, Sanjuan X, Closa A, et al. Exome sequencing reveals AMER1 as a frequently mutated gene in colorectal cancer. Clin Cancer Res. 2015; 21:4709-18. https://doi.org/10.1158/1078-0432.CCR-15-0159.

38. Cajuso T, Hanninen UA, Kondelin J, Gylfe AE, Tanskanen T, Katainen R, Pitkanen E, Ristolainen H, Kaasinen E, Taipale M, Taipale J, Bohm J, Renkonen-Sinisalo L, et al. Exome sequencing reveals frequent inactivating mutations in ARID1A, ARID1B, ARID2 and ARID4A in microsatellite unstable colorectal cancer. Int J Cancer. 2014; 135:611-23. https://doi.org/10.1002/ijc.28705.

39. Patsos G, Andre S, Roeckel N, Gromes R, Gebert J, Kopitz J, Gabius HJ. Compensation of loss of protein function in microsatellite-unstable colon cancer cells (HCT116): a gene-dependent effect on the cell surface glycan profile. Glycobiology. 2009; 19:726-34. https://doi.org/10.1093/glycob/cwp040.

40. Matsumoto A, Onoyama I, Nakayama KI. Expression of mouse Fbxw7 isoforms is regulated in a cell cycle- or p53dependent manner. Biochem Biophys Res Commun. 2006; 350:114-9. https://doi.org/10.1016/j.bbrc.2006.09.003.

41. Liu Y, Zhang X, Han C, Wan G, Huang X, Ivan C, Jiang D, Rodriguez-Aguayo C, Lopez-Berestein G, Rao PH, Maru DM, Pahl A, He X, et al. TP53 loss creates therapeutic vulnerability in colorectal cancer. Nature. 2015; 520:697701. https://doi.org/10.1038/nature14418.

42. Schubbert S, Shannon K, Bollag G. Hyperactive ras in developmental disorders and cancer. Nat Rev Cancer. 2007; 7:295-308. https://doi.org/10.1038/nrc2109.

43. Liu Y, Luan L, Wang X. A randomized Phase II clinical study of combining panitumumab and bevacizumab, plus irinotecan, 5-fluorouracil, and leucovorin (FOLFIRI) compared with FOLFIRI alone as second-line treatment for patients with metastatic colorectal cancer and KRAS mutation. Onco Targets Ther. 2015; 8:1061-8. https://doi.org/10.2147/OTT.S81442. 
44. Normanno N, Rachiglio AM, Lambiase $M$, Martinelli E, Fenizia F, Esposito C, Roma C, Troiani T, Rizzi D, Tatangelo F, Botti G, Maiello E, Colucci G, et al. Heterogeneity of KRAS, NRAS, BRAF and PIK3CA mutations in metastatic colorectal cancer and potential effects on therapy in the CAPRI GOIM trial. Ann Oncol. 2015; 26:1710-4. https://doi.org/10.1093/annonc/mdv176.

45. Di Nicolantonio F, Martini M, Molinari F, Sartore-Bianchi A, Arena S, Saletti P, De Dosso S, Mazzucchelli L, Frattini $\mathrm{M}$, Siena S, Bardelli A. Wild-type BRAF is required for response to panitumumab or cetuximab in metastatic colorectal cancer. J Clin Oncol. 2008; 26:5705-12. https://doi.org/10.1200/JCO.2008.18.0786.

46. Prahallad A, Sun C, Huang S, Di Nicolantonio F, Salazar R, Zecchin D, Beijersbergen RL, Bardelli A, Bernards R. Unresponsiveness of colon cancer to BRAF(V600E) inhibition through feedback activation of EGFR. Nature. 2012; 483:100-3. https://doi.org/10.1038/nature10868.

47. Palmirotta R, Ludovici G, De Marchis ML, Leone B, Formica V, Ettorre GM, Cavaliere F, Della-Morte D, Ferroni P, Roselli M, Guadagni F. A comprehensive procedural approach to genotyping KRAS and BRAF from paraffin embedded tissues for diagnostic purposes. In Vivo. 2012; 26:537-47.
48. Ogino S, Lochhead P, Giovannucci E, Meyerhardt JA, Fuchs CS, Chan AT. Discovery of colorectal cancer PIK3CA mutation as potential predictive biomarker: power and promise of molecular pathological epidemiology. Oncogene. 2014; 33:2949-55. https://doi.org/10.1038/onc.2013.244.

49. Rosty C, Young JP, Walsh MD, Clendenning M, Sanderson K, Walters RJ, Parry S, Jenkins MA, Win AK, Southey MC, Hopper JL, Giles GG, Williamson EJ, et al. PIK3CA activating mutation in colorectal carcinoma: associations with molecular features and survival. PLoS One. 2013; 8:e65479. https://doi.org/10.1371/journal.pone.0065479.

50. Kim E, Ilic N, Shrestha Y, Zou L, Kamburov A, Zhu C, Yang X, Lubonja R, Tran N, Nguyen C, Lawrence MS, Piccioni F, Bagul M, et al. Systematic functional interrogation of rare cancer variants identifies oncogenic alleles. Cancer Discov. 2016; 6:714-26. https://doi.org/10.1158/2159-8290.CD-16-0160.

51. Bastide P, Darido C, Pannequin J, Kist R, Robine S, MartyDouble C, Bibeau F, Scherer G, Joubert D, Hollande F, Blache P, Jay P. Sox9 regulates cell proliferation and is required for Paneth cell differentiation in the intestinal epithelium. J Cell Biol. 2007; 178:635-48. https://doi.org/10.1083/jcb.200704152. 